\title{
Quasiparticle GW calculations for solids, molecules, and two-dimensional materials
}

\author{
Hüser, Falco; Olsen, Thomas; Thygesen, Kristian Sommer
}

Published in:

Physical Review B

Link to article, DOI:

10.1103/PhysRevB.87.235132

Publication date:

2013

Document Version

Publisher's PDF, also known as Version of record

Link back to DTU Orbit

Citation (APA):

Hüser, F., Olsen, T., \& Thygesen, K. S. (2013). Quasiparticle GW calculations for solids, molecules, and twodimensional materials. Physical Review B, 87(23), [235132]. https://doi.org/10.1103/PhysRevB.87.235132

\section{General rights}

Copyright and moral rights for the publications made accessible in the public portal are retained by the authors and/or other copyright owners and it is a condition of accessing publications that users recognise and abide by the legal requirements associated with these rights.

- Users may download and print one copy of any publication from the public portal for the purpose of private study or research.

- You may not further distribute the material or use it for any profit-making activity or commercial gain

- You may freely distribute the URL identifying the publication in the public portal

If you believe that this document breaches copyright please contact us providing details, and we will remove access to the work immediately and investigate your claim. 


\title{
Quasiparticle GW calculations for solids, molecules, and two-dimensional materials
}

\author{
Falco Hüser, ${ }^{1, *}$ Thomas Olsen, ${ }^{1}$ and Kristian S. Thygesen ${ }^{1,2, \dagger}$ \\ ${ }^{1}$ Center for Atomic-scale Materials Design (CAMD), Department of Physics and \\ Technical University of Denmark, 2800 Kgs. Lyngby, Denmark \\ ${ }^{2}$ Center for Nanostructured Graphene (CNG), and Technical University of Denmark, 2800 Kgs. Lyngby, Denmark
}

(Received 25 February 2013; revised manuscript received 23 May 2013; published 24 June 2013)

\begin{abstract}
We present a plane-wave implementation of the $\mathrm{G}_{0} \mathrm{~W}_{0}$ approximation within the projector augmented wave method code GPAW. The computed band gaps of ten bulk semiconductors and insulators deviate on average by $0.2 \mathrm{eV}(\sim 5 \%)$ from the experimental values, the only exception being $\mathrm{ZnO}$ where the calculated band gap is around $1 \mathrm{eV}$ too low. Similar relative deviations are found for the ionization potentials of a test set of 32 small molecules. The importance of substrate screening for a correct description of quasiparticle energies and Fermi velocities in supported two-dimensional (2D) materials is illustrated by the case of graphene/ $h$-BN interfaces. Due to the long-range Coulomb interaction between periodically repeated images, the use of a truncated interaction is found to be essential for obtaining converged results for 2D materials. For all systems studied, a plasmon-pole approximation is found to reproduce the full frequency results to within $0.2 \mathrm{eV}$ with a significant gain in computational speed. Throughout, we compare the $\mathrm{G}_{0} \mathrm{~W}_{0}$ results with different exact exchange-based approximations. For completeness, we provide a mathematically rigorous and physically transparent introduction to the notion of quasiparticle states.
\end{abstract}

DOI: 10.1103/PhysRevB.87.235132

PACS number(s): 71.10.-w, 71.15.Mb, 71.20.Nr, 33.15.Ry

\section{INTRODUCTION}

For several decades, density functional theory (DFT) $)^{1,2}$ has been the method of choice for electronic structure calculations due to its unique compromise between accuracy and efficiency. Large efforts have been made to develop better exchangecorrelation (xc) functionals continuously pushing the quality of total energy calculations towards the limit of chemical accuracy. However, it is well known that the Kohn-Sham singleparticle energies do not correspond to physical excitation energies, and in fact the widely used semilocal xc potentials significantly underestimate quasiparticle (QP) energy gaps. ${ }^{3,4}$ For molecules and insulators better results can be obtained by replacing a fraction of the local exchange potential with the nonlocal Hartree-Fock exchange potential, as in the hybrid functionals. In the range-separated hybrids, the nonlocal exchange is used only for the short-range part of the potential. This improves the quality of semiconductor band structures and leads to faster convergence with $k$-point sampling, albeit at the cost of introducing an empirical cutoff radius. Still, the (range-separated) hybrids tend to underestimate the role of exchange in systems with weak screening, such as low-dimensional structures, and fail to account correctly for the spatial variation in the screening at metal-insulator interfaces (see below).

Many-body perturbation theory, on the other hand, offers a powerful and rigorous framework for the calculation of quasiparticle (QP) excitations. The key quantity is the electronic self-energy which is an energy-dependent and spatially nonlocal analogous of the xc potential of DFT. The self-energy can be systematically approximated by summing certain classes of perturbation terms to infinite order in the Coulomb interaction. The GW approximation ${ }^{5}$ is the simplest approximation of this kind where the self-energy, $\Sigma$, is expanded to first order in the screened interaction. Symbolically it takes the form $\Sigma_{x c}=i G W$, where $G$ is the Green's function and $W=\varepsilon^{-1} V$, is the screened interaction. Comparing the GW self-energy to the exchange potential, which can be written as $V_{x}=i G V$, we see that the GW self-energy is essentially a dynamically screened version of the exchange potential.

Apart from screening the static exchange potential, the replacement of the bare Coulomb interaction by the dynamically screened potential introduces correlation effects, which accounts for the interaction of an electron (or a hole) with the polarization charge that it induces in the medium. This is a highly nonlocal effect that becomes particularly evident at metal/insulator interfaces such as a molecule on a metal surface or the graphene $/ h-\mathrm{BN}$ interfaces studied in the present work. For these systems, the correlation takes the form of an image charge effect that reduces the energy gap of the molecule or insulator by up to several electron volts. ${ }^{6-11}$

The GW approximation has been applied with great success to a broad class of materials ranging from bulk insulators, semiconductors, and metals to low-dimensional systems such as nanoclusters, surfaces and molecules (see, e.g., the reviews of Refs. 12-14). Beyond the calculation of QP energies, the GW method also serves as starting point for the calculation of optical spectra from the Bethe-Salpeter equation (BSE) $)^{15-18}$ and for quantitatively accurate modeling of electron transport at metal-molecule interfaces where the alignment of the molecular energy levels with the metal Fermi level is particularly important. ${ }^{19-23}$

In principle, the GW self-energy should be evaluated self-consistently. However, due to the computational demands of such an approach, nonselfconsistent $\left(\mathrm{G}_{0} \mathrm{~W}_{0}\right)$ calculations with the initial $G_{0}$ obtained from the local density approximation (LDA) or similar, have traditionally been preferred. ${ }^{24}$ Recently, fully self-consistent GW calculations have been performed for molecular systems yielding energies for the highest occupied orbitals with an absolute deviation from experiments of around $0.5 \mathrm{eV}^{25,26} \mathrm{In}$ comparison, the standard $\mathrm{G}_{0} \mathrm{~W}_{0} @ \mathrm{LDA}$ approach was found to yield slightly lower 
accuracy, while better results were achieved when starting from Hartree-Fock or hybrid calculations. ${ }^{25-28}$ For solids, earlier studies yielded contradictory conclusions regarding the accuracy of self-consistent versus nonselfconsistent GW calculations. More recently, the quasiparticle self-consistent GW method, in which the self-energy is evaluated with a self-consistently determined single-particle Hamiltonian, has been shown to yield excellent results for solids. ${ }^{29-32}$

On the practical side, any implementation of the GW approximation has to deal with similar numerical challenges. In addition to the already mentioned $\mathrm{G}_{0} \mathrm{~W}_{0}$ approximation, it is common practice to evaluate the $\mathrm{QP}$ energies using first-order perturbation theory starting from the Kohn-Sham eigenvalues thereby avoiding the calculation of off-diagonal matrix elements of the self-energy. This approach is based on the assumption that the QP wave functions are similar to the Kohn-Sham wave functions. As recently shown for a metal-molecule interface this is sometimes far from being the case. ${ }^{33}$ Another common simplification is the use of a plasmon pole approximation (PPA) for the dielectric function. The PPA leads to a considerable gain in efficiency by removing the need for evaluating the dielectric function at all frequency points and allowing the frequency convolution of $G$ and $W$ in the GW self-energy to be carried out analytically. In his original paper, Hedin introduced a static Coulomb hole and screened exchange (COHSEX) approximation to the full GW self-energy. The COHSEX approximation is computationally efficient and clearly illustrates the physics described by the GW approximation. However, its validity is limited to rather special cases and it should generally not be used for quantitative calculations.

In this paper we document the implementation of the $\mathrm{G}_{0} \mathrm{~W}_{0}$ method in the GPAW open source electronic structure code. ${ }^{34}$ GPAW is based on the projector augmented wave (PAW) method ${ }^{35,36}$ and supports both real space grid and plane wave representation for high accuracy as well as numerical atomic orbitals (LCAO) for high efficiency. The $\mathrm{G}_{0} \mathrm{~W}_{0}$ implementation is based on plane waves. The implementation supports both full frequency dependence (along the real axis) as well as the plasmon-pole approximation of Godby and Needs. ${ }^{37}$ For low-dimensional systems, in particular two-dimensional (2D) systems, a truncated Coulomb interaction should be used to avoid the long-range interactions between periodically repeated unit cells. For both solids, molecules and 2D systems, we find that the PPA gives excellent results with significant reduction of the computational efforts. In contrast, the static COHSEX and the PBE0 hybrid yield unsatisfactory results. An interesting alternative to $\mathrm{GW}$ is offered by the local, orbital dependent potential of Gritsenko, Leeuwen, Lenthe, and Baerends with the modifications from Kuisma (GLLBSC), which explicitly adds the derivative discontinuity to the Kohn-Sham energy gap. ${ }^{38}$ The GLLBSC band gaps for solids are found to lie on average within $0.4 \mathrm{eV}$ of the $\mathrm{G}_{0} \mathrm{~W}_{0}$ values but give similar accuracy when compared to experimental data. The GLLBSC ionization potentials of molecules are in average $1.5 \mathrm{eV}$ below the $\mathrm{G}_{0} \mathrm{~W}_{0}$ values.

The paper is organized as follows. Sec. II gives a general introduction to the theory of quasiparticle states. In Sec. III, we briefly review the central equations of the $\mathrm{G}_{0} \mathrm{~W}_{0}$ method in a plane-wave basis and discuss some details of our implementation. In Sec. IV, we present results for bulk semiconductors, insulators and metals, comparing with experiments and previous calculations. The application to $2 \mathrm{D}$ systems is illustrated in Sec. V by the example of graphene on hexagonal boron nitride and the importance of screening effects on the QP energies is discussed. Finally, we test the implementation on finite systems by calculating the ionization potential of a set of 32 small molecules in Sec. VI.

\section{QUASIPARTICLE THEORY}

Quasiparticle states provide a rigorous generalization of the concept of single-particle orbitals to interacting electron systems. In this section we provide a compact, self-contained introduction to the general theory of quasiparticle states with a combined focus on physical interpretation and mathematical rigor. This presentation is completely formal; in particular we shall not discuss the physics and computation of specific self-energy approximations. Our presentation is thus complementary to most other papers on the GW method which tend to focus on the theory and derivation of the GW self-energy within the framework of many-body Green's function theory. To avoid inessential mathematical complications, we shall make the assumption that the system under consideration is finite and the relevant excitations are discrete.

\section{A. Definition of QP energies and wave functions}

We denote the $N$-particle many-body eigenstates and energies by $\left|\Psi_{i}^{N}\right\rangle$ and $E_{i}^{N}$, respectively. The occupied and unoccupied QP orbitals are denoted $\left|\psi_{i-}^{\mathrm{QP}}\right\rangle$ and $\left|\psi_{i+}^{\mathrm{QP}}\right\rangle$, respectively. These belong to the single-particle Hilbert space and are defined as:

$$
\begin{aligned}
& \psi_{i-}^{\mathrm{QP}}(\mathbf{r})^{*}=\left\langle\Psi_{i}^{N-1}|\hat{\Psi}(\mathbf{r})| \Psi_{0}^{N}\right\rangle \\
& \psi_{i+}^{\mathrm{QP}}(\mathbf{r})=\left\langle\Psi_{i}^{N+1}\left|\hat{\Psi}^{\dagger}(\mathbf{r})\right| \Psi_{0}^{N}\right\rangle
\end{aligned}
$$

where $\hat{\Psi}(\mathbf{r})$ and $\hat{\Psi}^{\dagger}(\mathbf{r})$ are the field operators annihilating and creating an electron at point $\mathbf{r}$, respectively. The QP wave functions defined above are also sometimes referred to as Lehman amplitudes or Dyson orbitals.

The corresponding QP energies are defined by

$$
\begin{aligned}
& \varepsilon_{i-}^{\mathrm{QP}}=E_{0}^{N}-E_{i}^{N-1} \\
& \varepsilon_{i+}^{\mathrm{QP}}=E_{i}^{N+1}-E_{0}^{N} .
\end{aligned}
$$

They represent the excitation energies of the $(N \pm 1)$-particle system relative to $E_{0}^{N}$ and thus correspond to electron addition and removal energies. It is clear that $\varepsilon_{i+}^{\mathrm{QP}}>\mu$ while $\varepsilon_{i-}^{\mathrm{QP}} \leqslant \mu$ where $\mu$ is the chemical potential. Having noted this, we can in fact drop the $+/-$ subscripts on the QP states and energies. We shall do that in most of the following to simplify the notation.

The fundamental energy gap is defined as

$$
\begin{aligned}
E_{\mathrm{gap}} & =\varepsilon_{0+}^{\mathrm{QP}}-\varepsilon_{0-}^{\mathrm{QP}} \\
& =E_{0}^{N+1}+E_{0}^{N-1}-2 E_{0}^{N} .
\end{aligned}
$$


We note that $E_{\text {gap }}$ can also be expressed within the framework of Kohn-Sham (KS) theory as

$$
E_{\text {gap }}=\varepsilon_{N+1}^{\mathrm{KS}}-\varepsilon_{N}^{\mathrm{KS}}+\Delta_{x c},
$$

where $\varepsilon_{n}^{\mathrm{KS}}$ are the (exact) Kohn-Sham energies and $\Delta_{x c}$ is the derivative discontinuity. ${ }^{39}$

\section{B. Interpretation of QP wave functions}

Since the many-body eigenstates of an interacting electron system are not Slater determinants, the notion of single-particle orbitals is not well defined a priori. For weakly correlated systems we can, however, expect that the single-particle picture applies to a good approximation. To make this precise we ask to what extent the state $\left|\Psi_{i}^{N+1}\right\rangle$ can be regarded as a single-particle excitation from the ground state, i.e., to what extent it can be written on the form $c_{\phi}^{\dagger}\left|\Psi_{0}^{N}\right\rangle$ when $\phi$ is chosen in an optimal way. It turns out that the optimal $\phi$ is exactly the QP orbital. This statement follows simply from the observation ${ }^{80}$

$$
\left\langle\phi \mid \psi_{i+}^{\mathrm{QP}}\right\rangle=\left\langle\Psi_{i}^{N+1}\left|\hat{c}_{\phi}^{\dagger}\right| \Psi_{0}^{N}\right\rangle,
$$

for any orbital $\phi$. Similarly, $\left|\psi_{i}^{-}\right\rangle$is the orbital that makes $\hat{c}_{\phi}\left|\Psi_{0}^{N}\right\rangle$ the best approximation to the excited state $\left|\Psi_{i}^{N-1}\right\rangle$. Consequently, the QP wave function $\psi_{i \pm}^{\mathrm{QP}}$ is the single-particle orbital that best describes the state of the extra electron/hole in the excited state $\left|\Psi_{i}^{N \pm 1}\right\rangle$.

From Eq. (8) it follows that the norm of a QP orbital is a measure of how well the true excitation can be described as a single-particle excitation. Precisely,

$$
\left.\left\|\psi_{i+}^{\mathrm{QP}}\right\|=\max _{\phi}\left\{\left\langle\Psi_{i}^{N+1}\left|\hat{c}_{\phi}^{\dagger}\right| \Psi_{0}^{N}\right\rangle,\|\phi\|=1\right\rangle\right\},
$$

and similarly for the norm of $\psi_{i-}^{\mathrm{QP}}$.

The definition (1) implies a one-to-one correspondence between QP states and the excited many-body states $\left|\Psi_{i}^{N \pm 1}\right\rangle$. Obviously, most of the latter are not even approximately of the single-particle type. These are characterized by a vanishing (or very small) norm of the corresponding $\mathrm{QP}$ orbital. In case of noninteracting electrons the QP states have norms 1 or 0 . The former correspond to single excitations (Slater determinants) of the form $c_{n}^{\dagger}\left|\Psi_{0}^{N}\right\rangle$ while the latter correspond to multiple particle excitations, e.g., $c_{n}^{\dagger} c_{m}^{\dagger} c_{k}\left|\Psi_{0}^{N}\right\rangle$. Strictly speaking the term quasiparticle should be used only for those $\left|\psi_{i}^{\mathrm{QP}}\right\rangle$ whose norm is close to 1 . The number of such states and whether any exists at all, depends on the system. For weakly correlated systems, one can expect a one-to-one correspondence between the QP states with norm $\sim 1$ and the single-particle states of some effective noninteracting Hamiltonian, at least for the low-lying excitations.

\section{Quasiparticle equation and self-energy}

Below we show that QP states fulfill a generalized eigenvalue equation known as the QP equation, and we derive a useful expression for the norm of a QP state in terms of the self-energy.
The QP states and energies are linked to the single-particle Green's function via the Lehmann spectral representation ${ }^{40}$

$$
G(z)=\sum_{i} \frac{\left|\psi_{i}^{\mathrm{QP}}\right\rangle\left\langle\psi_{i}^{\mathrm{QP}}\right|}{z-\varepsilon_{i}^{\mathrm{QP}}},
$$

where $z$ is a complex number and it is understood that the sum runs over both occupied and unoccupied QP states. It follows that $G(z)$ is analytic in the entire complex plane except for the real points $\varepsilon_{i}^{\mathrm{QP}}$, which are simple poles. We note in passing that $G(z)$ equals the Fourier transform of the retarded (advanced) Green's function in the upper (lower) complex half plane.

The Green's function also satisfies the Dyson equation

$$
G(z)=\left[z-H_{0}-\Sigma_{x c}(z)\right]^{-1},
$$

where $H_{0}$ is the noninteracting part of the Hamiltonian including the Hartree field and $\Sigma_{x c}$ is the exchange-correlation self-energy. The Dyson equation can be derived using manybody perturbation theory or it can simply be taken as the definition of the self-energy operator.

In the case where $\varepsilon_{i}^{\mathrm{QP}}$ belongs to the discrete spectrum, $\psi_{i}^{\mathrm{QP}}$ and $\varepsilon_{i}^{\mathrm{QP}}$ are solutions to the $\mathrm{QP}$ equation

$$
\left[H_{0}+\Sigma_{x c}\left(\varepsilon_{i}^{\mathrm{QP}}\right)\right]\left|\psi_{i}^{\mathrm{QP}}\right\rangle=\varepsilon_{i}^{\mathrm{QP}}\left|\psi_{i}^{\mathrm{QP}}\right\rangle .
$$

This follows from the residue theorem by integrating the equation $\left[z-H_{0}-\Sigma_{x c}(z)\right] G(z)=1$ along a complex contour enclosing the simple pole $\varepsilon_{i}^{\mathrm{QP}}$.

The operator $\left[H_{0}+\Sigma_{x c}(z)\right]$ is non-Hermitian and is diagonalized by a set of nonorthogonal eigenvectors,

$$
\left[H_{0}+\Sigma_{x c}(z)\right]\left|\psi_{n}(z)\right\rangle=\varepsilon_{n}(z)\left|\psi_{n}(z)\right\rangle .
$$

Using these eigenvectors, the GF can be expressed in an alternative spectral form

$$
G(z)=\sum_{n} \frac{\left|\psi_{n}(z)\right\rangle\left\langle\psi^{n}(z)\right|}{z-\varepsilon_{n}(z)} .
$$

where $\left\{\psi^{n}(z)\right\}$ is the dual basis of $\left\{\psi_{n}(z)\right\}$, which by definition satisfies $\left\langle\psi_{n}(z) \mid \psi^{m}(z)\right\rangle=\delta_{n m}{ }^{81}$ We shall take the functions $\psi_{n}(z)$ to be normalized which also fixes the normalization of the dual basis.

In general, the vectors $\psi_{n}(z)$ do not have any physical meaning but are pure mathematical objects. An exception occurs for $z=\varepsilon_{i}^{\mathrm{QP}}$ where one of the vectors $\psi_{n}\left(\varepsilon_{i}^{\mathrm{QP}}\right)$ coincide with the QP orbital $\psi_{i}^{\mathrm{QP}}$ (except for normalization). We shall denote that vector by $\psi_{i}\left(\varepsilon_{i}^{\mathrm{QP}}\right)$, i.e.,

$$
\left|\psi_{i}\left(\varepsilon_{i}^{\mathrm{QP}}\right)\right\rangle=\left|\psi_{i}^{\mathrm{QP}}\right\rangle /\left\|\psi_{i}^{\mathrm{QP}}\right\| .
$$

By equating the matrix element $\left\langle\psi^{i}(z)|G(z)| \psi_{i}(z)\right\rangle$ evaluated using the two alternative spectral representations Eq. (10) and Eq. (14), and integrating along a contour enclosing the pole $\varepsilon_{i}^{\mathrm{QP}}$, we obtain

$$
\left\langle\psi^{i}\left(\varepsilon_{i}^{\mathrm{QP}}\right) \mid \psi_{i}^{\mathrm{QP}}\right\rangle\left\langle\psi_{i}^{\mathrm{QP}} \mid \psi_{i}\left(\varepsilon_{i}^{\mathrm{QP}}\right)\right\rangle=\frac{1}{1-\varepsilon_{i}^{\prime}\left(\varepsilon_{i}^{\mathrm{QP}}\right)},
$$

where the prime denotes the derivative with respect to $z$. This result follows by application of the residue theorem. Using 
Eq. (15) it follows that the norm of the QP states is given by

$$
\begin{aligned}
\left\|\psi_{i}^{\mathrm{QP}}\right\|^{2} & =\left\langle\psi_{i}\left(\varepsilon_{i}^{\mathrm{QP}}\right)\left|1-\Sigma_{x c}^{\prime}\left(\varepsilon_{i}^{\mathrm{QP}}\right)\right| \psi_{i}\left(\varepsilon_{i}^{\mathrm{QP}}\right)\right\rangle^{-1} \\
& \equiv Z_{i},
\end{aligned}
$$

where we have used the Hellman-Feynman theorem to differentiate $\varepsilon_{i}(z)=\left\langle\psi_{i}(z)\left|H_{0}+\Sigma_{x c}(z)\right| \psi_{i}(z)\right\rangle$.

\section{Linearized QP equation}

Given a self-energy operator, one must solve the QP equation to obtain the QP states and energies. This is complicated by the fact that the self-energy must be evaluated at the QP energies, which are not known a priori. Instead, one can start from an effective noninteracting Hamiltonian (in practice often the Kohn-Sham Hamiltonian),

$$
\left[H_{0}+V_{x c}\right]\left|\psi_{i}^{s}\right\rangle=\varepsilon_{i}^{s}\left|\psi_{i}^{s}\right\rangle,
$$

and treat $\Sigma_{x c}\left(\varepsilon_{i}^{\mathrm{QP}}\right)-V_{x c}$ using first-order perturbation theory. Thus we write $\varepsilon_{i}^{\mathrm{QP}}=\varepsilon_{i}^{s}+\varepsilon_{i}^{(1)}$ with

$$
\begin{aligned}
\varepsilon_{i}^{(1)} & =\left\langle\psi_{i}^{s}\left|\Sigma_{x c}\left(\varepsilon_{i}^{\mathrm{QP}}\right)-V_{x c}\right| \psi_{i}^{s}\right\rangle \\
& =\left\langle\psi_{i}^{s}\left|\Sigma_{x c}\left(\varepsilon_{i}^{s}\right)+\left(\varepsilon_{i}^{\mathrm{QP}}-\varepsilon_{i}^{s}\right) \Sigma_{x c}^{\prime}\left(\varepsilon_{i}^{s}\right)-V_{x c}\right| \psi_{i}^{s}\right\rangle .
\end{aligned}
$$

Rearranging this equation yields

$$
\varepsilon_{i}^{\mathrm{QP}}=\varepsilon_{i}^{s}+Z_{i}^{s} \cdot\left\langle\psi_{i}^{s}\left|\Sigma_{x c}\left(\varepsilon_{i}^{s}\right)-V_{x c}\right| \psi_{i}^{s}\right\rangle,
$$

where

$$
Z_{i}^{s}=\left\langle\psi_{i}^{s}\left|1-\Sigma_{x c}^{\prime}\left(\varepsilon_{i}^{s}\right)\right| \psi_{i}^{s}\right\rangle^{-1}
$$

approximates the true QP norm.

If $Z_{i}^{s} \ll 1$ we can conclude that $\psi_{i}^{s}$ is not a (proper) QP state. There can be two reasons for this: (i) the electrons are strongly correlated and as a consequence the QP picture does not apply, or (ii) $\psi_{i}^{s}$ is not a good approximation to the true QP wave function $\psi_{i}^{\mathrm{QP}}$. While (i) is rooted in the physics of the underlying electron system, reason (ii) merely says that the Kohn-Sham orbital does not describe the true many-body excitations well. For an example where the QP picture is completely valid, i.e., all the QP states have norms very close to 1 or 0 , but where simple noninteracting orbitals do not provide a good approximation to them, we refer to Ref. 33 .

\section{III. $G_{0} W_{0}$ APPROXIMATION}

The self-energy of the GW approximation is given as a product of the (time-ordered) Green's function and screened Coulomb potential and can be split into an exchange and a correlation part, $\Sigma_{\mathrm{GW}}=V_{x}+\Sigma_{c}$, where $V_{x}$ is the nonlocal Hartree-Fock exchange potential. The correlation contribution (which we from now on refer to as the self-energy $\Sigma=\Sigma_{c}$ ) is then evaluated by introducing the difference between the screened and the bare Coulomb potential $\bar{W}=W-V$,

$$
\Sigma\left(\mathbf{r} t, \mathbf{r}^{\prime} t^{\prime}\right)=i G\left(\mathbf{r} t, \mathbf{r}^{\prime} t^{\prime}\right) \bar{W}\left(\mathbf{r} t, \mathbf{r}^{\prime} t^{\prime}\right),
$$

which becomes a convolution in frequency domain

$$
\Sigma\left(\mathbf{r}, \mathbf{r}^{\prime} ; \omega\right)=\frac{i}{2 \pi} \int d \omega^{\prime} G\left(\mathbf{r}, \mathbf{r}^{\prime} ; \omega+\omega^{\prime}\right) \bar{W}\left(\mathbf{r}, \mathbf{r}^{\prime} ; \omega^{\prime}\right) .
$$

In this way, the exchange and the correlation contributions can be treated separately at different levels of accuracy.
Additionally, the screened Coulomb potential approaches the bare one for large frequencies, so that $\bar{W}$ vanishes in this limit making the frequency integration numerically stable.

In the present $\mathrm{G}_{0} \mathrm{~W}_{0}$ approach, the self-energy is constructed from Kohn-Sham wave functions $|n \mathbf{k}\rangle$ and eigenvalues $\varepsilon_{n \mathbf{k}}^{s}$, where $n$ and $\mathbf{k}$ denote band and $k$-point index, respectively. Throughout this paper, spin indices are suppressed in order to simplify the notation.

Using the spectral representation for the Green's function in this basis and Fourier transforming to reciprocal space, the diagonal terms of the self-energy read ${ }^{41}$

$$
\begin{aligned}
\Sigma_{n \mathbf{k}} \equiv & \langle n \mathbf{k}|\Sigma(\omega)| n \mathbf{k}\rangle \\
= & \frac{1}{\Omega} \sum_{\mathbf{G G}^{\prime}} \sum_{\mathbf{q}}^{1 . \mathrm{BZ}} \sum_{m}^{\text {all }} \frac{i}{2 \pi} \int_{-\infty}^{\infty} d \omega^{\prime} \bar{W}_{\mathbf{G G}^{\prime}}\left(\mathbf{q}, \omega^{\prime}\right) \\
& \times \frac{\rho_{m \mathbf{k}-\mathbf{q}}^{n \mathbf{k}}(\mathbf{G}) \rho_{m \mathbf{k}-\mathbf{q}}^{n \mathbf{k} *}\left(\mathbf{G}^{\prime}\right)}{\omega+\omega^{\prime}-\varepsilon_{m \mathbf{k}-\mathbf{q}}^{s}+i \eta \operatorname{sgn}\left(\varepsilon_{m \mathbf{k}-\mathbf{q}}^{s}-\mu\right)},
\end{aligned}
$$

where $m$ runs over all bands, $\mathbf{q}$ covers the differences between all $k$ points in the first Brillouin zone. The infinitesimal $\eta \rightarrow 0^{+}$ ensures the correct time ordering of the Green's function, $\Omega=$ $\Omega_{\text {cell }} N_{\mathbf{k}}$ is the total crystal volume, and $\mu$ is the chemical potential. The pair density matrix elements are defined as:

$$
\rho_{m \mathbf{k}-\mathbf{q}}^{n \mathbf{k}}(\mathbf{G}) \equiv\left\langle n \mathbf{k}\left|e^{i(\mathbf{q}+\mathbf{G}) \mathbf{r}}\right| m \mathbf{k}-\mathbf{q}\right\rangle .
$$

The potential $\bar{W}_{\mathbf{G G}^{\prime}}(\mathbf{q}, \omega)$ is obtained from the symmetrized, time-ordered dielectric function in the random phase approximation (RPA)

$$
\bar{W}_{\mathbf{G G}^{\prime}}(\mathbf{q}, \omega)=\frac{4 \pi}{|\mathbf{q}+\mathbf{G}|}\left(\epsilon_{\mathbf{G G}^{\prime}}^{-1}(\mathbf{q}, \omega)-\delta_{\mathbf{G G}^{\prime}}\right) \frac{1}{\left|\mathbf{q}+\mathbf{G}^{\prime}\right|} .
$$

The calculation of the dielectric function in the GPAW code is described in Ref. 42.

The quasi-particle spectrum is then calculated with Eq. (22) using first-order perturbation theory in $\left(\Sigma_{\mathrm{GW}}-V_{x c}\right)$, where $V_{x c}$ is the Kohn-Sham exchange-correlation potential

$$
\varepsilon_{n \mathbf{k}}^{\mathrm{QP}}=\varepsilon_{n \mathbf{k}}^{s}+Z_{n \mathbf{k}}^{s} \operatorname{Re}\left\langle n \mathbf{k}\left|\Sigma\left(\varepsilon_{n \mathbf{k}}^{s}\right)+V_{x}-V_{x c}\right| n \mathbf{k}\right\rangle,
$$

with a renormalization factor given by

$$
Z_{n \mathbf{k}}^{s}=\left(1-\operatorname{Re}\left\langle n \mathbf{k}\left|\Sigma^{\prime}\left(\varepsilon_{n \mathbf{k}}^{s}\right)\right| n \mathbf{k}\right\rangle\right)^{-1},
$$

where the derivative of the self-energy with respect to the frequency is calculated analytically from Eq. (26). The calculation of the exact exchange potential within GPAW is described in Ref. 34 using the plane-wave expressions of Ref. 43.

As discussed in the previous section, this first-order approach, i.e., using only the diagonal terms of the self-energy, is based on the assumption that the true QP wave functions and energies are similar to the Kohn-Sham wave functions and energies. To proceed beyond this approximation one must evaluate also the off-diagonal terms of the self-energy and invoke (partial) self-consistency. This is, however, beyond the scope of the present work. Similarly, the effect of electronelectron interactions on the QP lifetimes, which in principle can be deduced from the imaginary part of the GW self-energy, will not be considered in this study. 


\section{A. Frequency grid}

For a fully frequency-dependent GW calculation, the dielectric matrix and thus the screened potential is evaluated on a user-defined grid of real frequencies and the integration in Eq. (26) is performed numerically. The frequency grid is chosen to be linear up to $\omega_{\text {lin }}$ with a spacing of $\Delta \omega$, which typically is set to $0.05 \mathrm{eV}$. Above $\omega_{\text {lin }}$ the grid spacing grows linearly up to a maximum frequency, $\omega_{\max }$. In practice we set $\omega_{\max }$ to equal the maximum transition energy and $\omega_{\text {lin }} \approx(1 / 4) \cdot \omega_{\max }$ which results in a few thousand frequency points. Compared to a fully linear grid, the use of a nonuniform grid gives a computational speedup of around a factor 2-3 without any loss of accuracy. The broadening parameter $\eta$ is set to $4 \Delta \omega$ to ensure a proper resolution of all spectral features.

\section{B. Plasmon-pole approximation}

In the plasmon-pole approximation (PPA), the frequency dependence of the dielectric function $\epsilon_{\mathbf{G G}^{\prime}}^{-1}(\mathbf{q}, \omega)$ is modeled as a single-pole approximation

$$
\begin{aligned}
\varepsilon_{\mathbf{G G}^{\prime}}^{-1}(\mathbf{q}, \omega)= & R_{\mathbf{G G}^{\prime}}(\mathbf{q})\left(\frac{1}{\omega-\tilde{\omega}_{\mathbf{G G}^{\prime}}(\mathbf{q})+i \eta}\right. \\
& \left.-\frac{1}{\omega+\tilde{\omega}_{\mathbf{G G}^{\prime}}(\mathbf{q})-i \eta}\right) .
\end{aligned}
$$

The plasmon frequency $\tilde{\omega}_{\mathbf{G G}^{\prime}}(\mathbf{q})$ and the (real) spectral function $R_{\mathbf{G G}^{\prime}}(\mathbf{q})$ are determined by fitting this function to the dielectric matrix given at the frequency points $\omega_{1}=0$ and $\omega_{2}=i E_{0}$ :

$$
\begin{gathered}
\tilde{\omega}_{\mathbf{G G}^{\prime}}(\mathbf{q})=E_{0} \sqrt{\frac{\varepsilon_{\mathbf{G G}^{\prime}}^{-1}\left(\mathbf{q}, \omega_{2}\right)}{\varepsilon_{\mathbf{G G}^{\prime}}^{-1}\left(\mathbf{q}, \omega_{1}\right)-\varepsilon_{\mathbf{G G}^{\prime}}^{-1}\left(\mathbf{q}, \omega_{2}\right)},} \\
R_{\mathbf{G G}^{\prime}}(\mathbf{q})=-\frac{\tilde{\omega}_{\mathbf{G G}^{\prime}}(\mathbf{q})}{2} \varepsilon_{\mathbf{G G}^{\prime}}^{-1}\left(\mathbf{q}, \omega_{1}\right) .
\end{gathered}
$$

Using the relation

$$
\lim _{\eta \rightarrow 0^{+}} \frac{1}{x \pm i \eta}=\mathcal{P}\left\{\frac{1}{x}\right\} \mp i \pi \delta(x)
$$

where $\mathcal{P}$ denotes the Cauchy principal value, the spectral function of the screened potential, $\operatorname{Im}\left\{\bar{W}_{\mathbf{G G}^{\prime}}(\mathbf{q}, \omega)\right\}$, is simply a $\delta$ function at the plasmon frequencies $\pm \tilde{\omega}_{\mathbf{G G}^{\prime}}(\mathbf{q})$. Similarly, the relation (34) can be used in Eq. (26) allowing the GW self-energy to be evaluated analytically.

The PPA is expected to be a good approximation, when the overall structure of the dielectric function is dominated by a single (complex) pole. The true dielectric function will show variations on a finer scale. However, these are averaged out by the frequency integration in Eq. (26). In practice, we set the free parameter, $E_{0}$, to 1 Hartree in all our calculations and we find results to be insensitive to variations of around 0.5 Hartree.

\section{Static COHSEX}

By setting $\omega-\varepsilon_{m \mathbf{k}-\mathbf{q}}=0$ in Eq. (26), the self-energy becomes frequency independent and can be split into two parts, named Coulomb hole and screened exchange. ${ }^{44}$ The first term arises from the poles of the screened potential and describes the local interaction of an electron with its induced charge

$$
\Sigma^{\mathrm{COH}}=\frac{1}{2} \delta\left(\mathbf{r}-\mathbf{r}^{\prime}\right)\left[W\left(\mathbf{r}, \mathbf{r}^{\prime} ; \omega=0\right)-V\left(\mathbf{r}, \mathbf{r}^{\prime}\right)\right] .
$$

The plane-wave expression for a matrix element on a Bloch state $|n \mathbf{k}\rangle$ becomes

$$
\Sigma_{n \mathbf{k}}^{\mathrm{COH}}=\frac{1}{2 \Omega} \sum_{\mathbf{G G}^{\prime}} \sum_{\mathbf{q}} \sum_{m}^{\text {all }} \bar{W}_{\mathbf{G G}^{\prime}}(\mathbf{q}, 0) \rho_{m \mathbf{k}-\mathbf{q}}^{n \mathbf{k}}(\mathbf{G}) \rho_{m \mathbf{k}-\mathbf{q}}^{n \mathbf{k} *}\left(\mathbf{G}^{\prime}\right) .
$$

The second term originates from the poles of the Green's function and is identical to the exchange term in HartreeFock theory with the Coulomb kernel replaced by the screened interaction

$$
\Sigma^{\mathrm{SEX}}=-\sum_{j}^{\mathrm{occ}} \phi_{j}^{*}(\mathbf{r}) \phi_{j}\left(\mathbf{r}^{\prime}\right) W\left(\mathbf{r}, \mathbf{r}^{\prime} ; \omega=0\right),
$$

which yields the matrix element

$$
\Sigma_{n \mathbf{k}}^{\mathrm{SEX}}=-\frac{1}{\Omega} \sum_{\mathbf{G G}^{\prime}} \sum_{\mathbf{q}} \sum_{m}^{\mathrm{occ}} W_{\mathbf{G G}^{\prime}}(\mathbf{q}, 0) \rho_{m \mathbf{k}-\mathbf{q}}^{n \mathbf{k}}(\mathbf{G}) \rho_{m \mathbf{k}-\mathbf{q}}^{n \mathbf{k} *}\left(\mathbf{G}^{\prime}\right) .
$$

The quasiparticle energies are then given as

$$
\varepsilon_{n \mathbf{k}}^{\mathrm{QP}}=\varepsilon_{n \mathbf{k}}^{s}+\left\langle n \mathbf{k}\left|\Sigma^{\mathrm{SEX}}+\Sigma^{\mathrm{COH}}-V_{x c}\right| n \mathbf{k}\right\rangle .
$$

\section{Coulomb divergence}

For $\mathbf{q} \rightarrow 0$, the head, $\bar{W}_{\mathbf{0 0}}(\mathbf{q})$, and wings, $\bar{W}_{\mathbf{G 0}}(\mathbf{q}), \bar{W}_{\mathbf{0}} \mathbf{G}^{\prime}(\mathbf{q})$, of the screened potential diverge as $1 / q^{2}$ and $1 / q$, respectively. These divergences are, however, integrable. In the limit of a very fine $k$-point sampling we have $\sum_{\mathbf{q}} \rightarrow \frac{\Omega}{(2 \pi)^{3}} \int d q 4 \pi q^{2}$, and thus we can replace the $\mathbf{q}=0$ term in the $q$ sum of Eq. (26) by an integral over a sphere in reciprocal space with volume $\Omega_{\mathrm{BZ}} / N_{\mathbf{k}}$. The head and wings of the screened potential then take the form

$$
\begin{gathered}
\bar{W}_{\mathbf{0 0}}(\mathbf{q}=0, \omega)=\frac{2 \Omega}{\pi}\left(\frac{6 \pi^{2}}{\Omega}\right)^{1 / 3}\left[\varepsilon_{\mathbf{0 0}}^{-1}(\mathbf{q} \rightarrow 0, \omega)-1\right], \\
\bar{W}_{\mathbf{G 0}}(\mathbf{q}=0, \omega)=\frac{1}{|\mathbf{G}|} \frac{\Omega}{\pi}\left(\frac{6 \pi^{2}}{\Omega}\right)^{2 / 3} \varepsilon_{\mathbf{G 0}}^{-1}(\mathbf{q} \rightarrow 0, \omega),
\end{gathered}
$$

with the dielectric function evaluated in the optical limit. ${ }^{42}$

\section{E. Coulomb truncation}

In order to avoid artificial image effects in supercell calculations of systems, which are nonperiodic in one direction (2D systems), we follow Ref. 45 and cut off the Coulomb interaction by a step function in the nonperiodic direction ( $z$ axis)

$$
\tilde{v}^{2 D}(\mathbf{r})=\frac{\theta\left(R-\left|r_{z}\right|\right)}{|\mathbf{r}|},
$$

where $R$ is the truncation length. In reciprocal space, this becomes

$$
\tilde{v}^{2 D}(\mathbf{G})=\frac{4 \pi}{\mathbf{G}^{2}}\left[1+e^{-G_{\|} R}\left(\frac{G_{z}}{G_{\|}} \sin \left(G_{z} R\right)-\cos \left(\left|G_{z}\right| R\right)\right)\right],
$$


where $G_{\|}$and $G_{z}$ are the parallel and perpendicular components of $\mathbf{G}$, respectively. By setting $R$ to half the length of the unit cell in $z$ direction, this simplifies to ${ }^{46}$

$$
\tilde{v}^{2 D}(\mathbf{G})=\frac{4 \pi}{\mathbf{G}^{2}}\left(1-e^{-G_{\|} R} \cos \left(\left|G_{z}\right| R\right)\right) .
$$

Since Eq. (43) and thereby Eq. (44) are not well defined for $G_{\|} \rightarrow 0$, we have to evaluate these terms by numerical integration

$$
\tilde{v}^{2 \mathrm{D}}\left(G_{\|}=0\right)=\frac{1}{\Omega^{\prime}} \int_{\Omega^{\prime}} d \mathbf{q}^{\prime} \tilde{v}^{2 \mathrm{D}}\left(G_{z}+\mathbf{q}^{\prime}\right),
$$

where $\Omega^{\prime}$ is a small BZ volume around $G_{\|}=0$. This integral is well defined and converges easily for a fine grid $\mathbf{q}^{\prime}$ not containing the $\Gamma$ point.

We mention that other methods have been applied to correct for the spurious long-range interaction in GW calculations for surfaces. ${ }^{47,48}$

\section{F. Computational details}

The calculation of one matrix element of the self-energy of Eq. (26) scales as $N_{\omega} \times N_{b} \times N_{k}^{2} \times N_{G}^{2}$ with number of frequency points, bands, $k$ points, and plane waves, respectively. The code is parallelized over $\mathbf{q}$ vectors. For calculations including the $\Gamma$ point only, i.e., isolated systems, full parallelization over bands is used instead. Therefore, the computational time scales linearly with the number of cores. The screened potential $\bar{W}_{\mathbf{G G}^{\prime}}(\mathbf{q}, \omega)$ is evaluated separately for every $\mathbf{q}$ as an array in $\mathbf{G}, \mathbf{G}^{\prime}$ and $\omega$. For large numbers of plane waves and frequency points, this array can be distributed onto different cores, thus reducing the memory requirement on every core.

In practice, the use of the plasmon-pole approximation gives a computational speedup of a factor of 5-20 on average compared to a full frequency calculation. For both methods (PPA and full frequency integration), the computational time spent on the evaluation of the dielectric matrix and on the calculation of the quasiparticle spectrum from the screened potential is comparable.

\section{SOLIDS}

As a first application, we calculate the band structures of ten simple semiconductors and insulators ranging from $\mathrm{Si}$ to $\mathrm{LiF}$ thus covering a broad range of band gap sizes of both direct and indirect nature. We compare the different approximation schemes within nonselfconsistent GW, namely (i) full frequency dependence, (ii) plasmon-pole approximation, and (iii) static COHSEX. In all these cases the self-energy is calculated with orbitals and single-particle energies obtained from an LDA calculation, i.e., $\mathrm{G}_{0} \mathrm{~W}_{0} @$ LDA. In addition we perform nonselfconsistent Hartree-Fock (HF), as well as PBE0 hybrid calculations in both cases using LDA orbitals. Finally, we compare to self-consistent GLLBSC ${ }^{38,49}$ calculations. The GLLBSC is based on the PBEsol correlation potential and uses an efficient approximation to the exact exchange optimized effective potential which allows for explicit evaluation of the derivative discontinuity, $\Delta_{x c}$. We have recently applied the GLLBSC in computational screening studies of materials for photocatalytic water splitting. ${ }^{50,51}$ Here we present a systematic assessment of its performance by comparing to experi-
TABLE I. Geometric structures.

\begin{tabular}{lcc}
\hline \hline & Structure & Lattice constant in $\AA$ \\
\hline $\mathrm{Si}$ & Diamond & 5.431 \\
$\mathrm{InP}$ & Zincblende & 5.869 \\
$\mathrm{GaAs}$ & Zincblende & 5.650 \\
$\mathrm{AlP}$ & Zincblende & 5.451 \\
$\mathrm{ZnO}$ & Zincblende & 4.580 \\
$\mathrm{ZnS}$ & Zincblende & 5.420 \\
$\mathrm{C}$ & Diamond & 3.567 \\
$\mathrm{BN}$ & Zincblende & 3.615 \\
$\mathrm{MgO}$ & Rocksalt & 4.212 \\
$\mathrm{LiF}$ & Rocksalt & 4.024 \\
\hline \hline
\end{tabular}

ments and GW results for various types of systems. The bulk structures and the used lattice constants are listed in Table I.

All calculations were performed with the GPAW code, which is based on the projector augmented wave method and supports both real space and plane-wave representations. In the present work only the plane-wave basis set has been used. The same set of parameters is used for the calculation of the dielectric matrix and the self-energy. For all GW calculations, convergence with respect to the plane wave cutoff, number of unoccupied bands and $k$ points has been tested carefully, together with the size of the frequency grid for the full frequency calculations. As an example, Fig. 1 shows the dependence of the $G_{0} W_{0}$ band gap of zinc oxide on the plane-wave cutoff and the number of $k$ points. For cutoff energies above $100 \mathrm{eV}$ (corresponding to around 200 plane waves and bands), the value of the band gap is converged to within $0.02 \mathrm{eV}$, whereas increasing the number of $k$ points results in a constant shift. For all the solids we have investigated, the band gap is well converged with $E_{\text {cut }}=$ 200-300 eV and a few hundred empty bands. For materials with direct band gaps $(9 \times 9 \times 9) k$ points were found to be sufficient, whereas for $\mathrm{AlP}, \mathrm{BN}, \mathrm{C}, \mathrm{Si}$, and $\mathrm{ZnS}$, which have indirect gaps, $(15 \times 15 \times 15) k$ points were used in order to clearly resolve the conduction band minimum.

The results for the band gaps are summarized in Fig. 2 and Table II along with experimental data. The last row shows

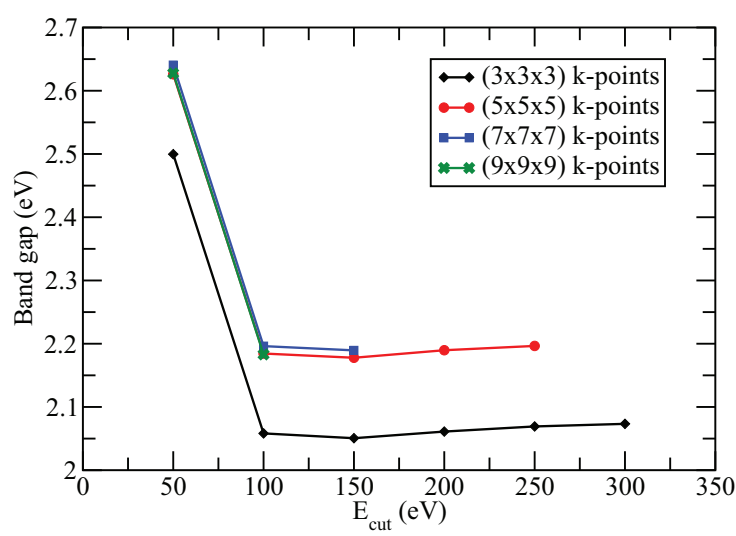

FIG. 1. (Color online) Convergence of the band gap of zinc oxide for $\mathrm{G}_{0} \mathrm{~W}_{0} @ \mathrm{LDA}$ with the plasmon-pole approximation. The number of bands is chosen equally to the number of plane waves corresponding to the respective cutoff energy; for example $300 \mathrm{eV}$ corresponds to $\sim 1100$ plane waves and bands. 


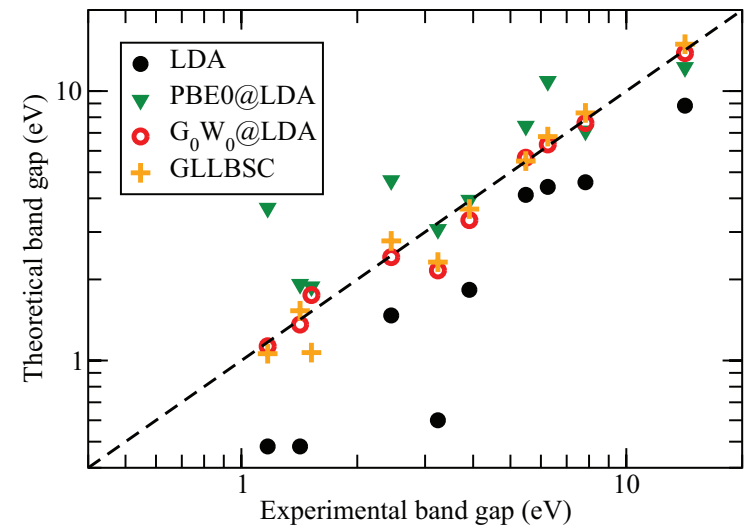

FIG. 2. (Color online) Comparison of calculated and experimental band gaps for the solids listed in Table I. The numerical values are listed in Table II. A logarithmic scale is used for better visualization. $\mathrm{G}_{0} \mathrm{~W}_{0} @ \mathrm{LDA}$ refers to the fully frequency-dependent nonselfconsistent GW based on LDA. The PBE0 results are obtained nonselfconsistently using LDA orbitals.

the mean absolute errors (MAE) of each method relative to experiment.

As expected LDA predicts much too small band gaps with relative errors as large as $400 \%$ in the case of GaAs. In contrast HF greatly overestimates the band gap for all systems yielding even larger relative errors than LDA and with absolute errors exceeding $7 \mathrm{eV}$. The failure of HF is particularly severe for systems with narrow band gaps like $\mathrm{Si}$ and $\mathrm{InP}$ where the relative error is up to $500 \%$ whereas the error for the large gap insulator $\mathrm{LiF}$ is $50 \%$. This difference can be understood from the relative importance of screening (completely neglected in HF) in the two types of systems. The PBE0 results lie in between LDA and HF with band gaps lying somewhat closer to the experimental values, however, still significantly overestimating the size of the gap for systems with small to intermediate band gap.

The inclusion of static screening within the COHSEX approximation significantly improves the bare HF results. However, with a MAE of $1.59 \mathrm{eV}$, the results are still unsatisfactory and there seems to be no systematic trend in the deviations from experiments, except for a slightly better performance for materials with larger band gaps. We mention that a detailed discussion of the drawbacks of COHSEX and how to correct its main deficiencies can be found in Ref. 52. In Ref. 53, the static COHSEX approximation was explored as a starting point for $\mathrm{G}_{0} \mathrm{~W}_{0}$ calculations and compared to quasiparticle self-consistent GW calculations. However, no systematic improvement over the LDA starting point was found.

Introducing dynamical screening in the self-energy brings the band gaps much closer to the experimental values. The $\mathrm{G}_{0} \mathrm{~W}_{0}$ calculations with the PPA and full frequency dependence yield almost identical results, with only small deviations of about $0.2 \mathrm{eV}$ for the large band gap systems $\mathrm{LiF}$ and $\mathrm{MgO}$, where the fully frequency-dependent method performs slightly better.

Our results agree well with previous works for $\mathrm{G}_{0} \mathrm{~W}_{0}$ calculations using $\mathrm{LDA}^{54}$ and $\mathrm{PBE}^{29}$ as starting points with mean absolute errors of 0.31 and $0.21 \mathrm{eV}$ in comparison, respectively. Compared to Ref. 29, the only significant deviations can be seen for GaAs and the wide gap systems, where our calculated band gaps are somewhat larger. We expect that this is due to the difference between LDA and PBE as starting point. The values reported in Ref. 54 are all smaller than ours. A more detailed comparison is, however, complicated because of the differences in the implementations: Ref. 54 uses a mixed basis set in an all-electron linear muffin-tin orbital (LMTO) framework. We note that for $\mathrm{LiF}$, the calculated band gap is

TABLE II. Band gaps in eV. The type of gap is indicated in the last column. The last row gives the mean absolute error compared to experiment. Experimental data is taken from Ref. 59. Note that the experimental data for $\mathrm{ZnO}$ refers to the wurtzite structure. We find the calculated band gap to be around $0.1 \mathrm{eV}$ smaller in the zincblende than in the wurtzite structure for both LDA, $_{0} \mathrm{~W}_{0}$ and $\mathrm{GLLBSC}_{\text {. }}$ Experimental gap for InP taken from Ref. 63.

\begin{tabular}{|c|c|c|c|c|c|c|c|c|c|}
\hline & \multirow[b]{2}{*}{ LDA } & \multirow[b]{2}{*}{ HF@LDA } & \multirow[b]{2}{*}{ PBE0@LDA } & \multicolumn{3}{|c|}{$\mathrm{G}_{0} \mathrm{~W}_{0} @ \mathrm{LDA}$} & \multirow[b]{2}{*}{ GLLBSC } & \multirow[b]{2}{*}{ Experiment } & \\
\hline & & & & COHSEX & PPA & dyn & & & \\
\hline $\mathrm{Si}$ & 0.48 & 5.26 & 3.68 & 0.56 & 1.09 & 1.13 & 1.06 & 1.17 & Indirect \\
\hline $\mathrm{InP}$ & 0.48 & 5.51 & 1.92 & $1.99^{\mathrm{a}}$ & 1.38 & 1.36 & 1.53 & 1.42 & Direct \\
\hline GaAs & 0.38 & 5.46 & $1.88^{\mathrm{b}}$ & $3.77^{\mathrm{c}}$ & 1.76 & 1.75 & 1.07 & 1.52 & Direct \\
\hline $\mathrm{AlP}$ & 1.47 & 7.15 & 4.66 & 1.88 & 2.38 & 2.42 & 2.78 & 2.45 & Indirect \\
\hline $\mathrm{ZnO}$ & 0.60 & $10.42^{\mathrm{d}}$ & $3.07^{\mathrm{e}}$ & 0.10 & 2.20 & 2.24 & 2.32 & 3.44 & Direct \\
\hline $\mathrm{ZnS}$ & 1.83 & 9.43 & $3.94^{\mathrm{f}}$ & 1.52 & 3.28 & 3.32 & 3.65 & 3.91 & Direct \\
\hline $\mathrm{C}$ & 4.12 & 11.83 & 7.42 & 6.51 & 5.59 & 5.66 & 5.50 & 5.48 & Indirect \\
\hline $\mathrm{BN}$ & 4.41 & 13.27 & 10.88 & 7.08 & 6.30 & 6.34 & 6.78 & 6.25 & Indirect \\
\hline $\mathrm{MgO}$ & 4.59 & 14.84 & 7.12 & 10.30 & 7.44 & 7.61 & 8.30 & 7.83 & Direct \\
\hline $\mathrm{LiF}$ & 8.83 & 21.86 & 12.25 & 16.02 & 13.64 & 13.84 & 14.93 & 14.20 & Direct \\
\hline$\overline{\mathrm{MAE}}$ & 2.05 & 5.74 & 1.52 & 1.59 & 0.35 & 0.31 & 0.41 & & \\
\hline
\end{tabular}

${ }^{\mathrm{a}} \mathrm{COHSEX}$ predicts an indirect band gap of $1.73 \mathrm{eV}$.

${ }^{b}$ PBE0 predicts an indirect band gap of $1.79 \mathrm{eV}$.

${ }^{\mathrm{c}} \mathrm{COHSEX}$ predicts an indirect band gap of $1.07 \mathrm{eV}$.

${ }^{\mathrm{d}} \mathrm{HF}$ predicts an indirect band gap of $9.73 \mathrm{eV}$.

${ }^{\text {e}} \mathrm{PBE0}$ predicts an indirect band gap of $2.83 \mathrm{eV}$.

${ }^{\mathrm{f}} \mathrm{PBE} 0$ predicts an indirect band gap of $3.80 \mathrm{eV}$. 
strongly dependent on the lattice constant. With only a slightly smaller lattice constant of $3.972 \AA$, which is the experimental value corrected for zero-point anharmonic expansion effects, ${ }^{55}$ the quasiparticle gap increases by $0.4 \mathrm{eV}$.

One well-known problematic case for the GW approximation is $\mathrm{ZnO}$ (both in the zincblende and the wurtzite structure). The calculated band gap in the present study at the $\mathrm{G}_{0} \mathrm{~W}_{0} @ \mathrm{LDA}$ level is about $1 \mathrm{eV}$ too low, which is consistent with other previous $\mathrm{G}_{0} \mathrm{~W}_{0}$ studies. ${ }^{56-59}$ Recent $\mathrm{G}_{0} \mathrm{~W}_{0}$ calculations employing pseudopotentials and the PPA ${ }^{60}$ as well as all-electron $\mathrm{G}_{0} \mathrm{~W}_{0}{ }^{61}$ have attributed this discrepancy to a very slow convergence of the band gap with respect to the number of bands. This is, however, not in agreement with our PAW-based calculations, which are well converged with a cutoff energy of $100 \mathrm{eV}$ and around 200 bands. We note that semicore $d$ states of zinc are explicitly included in our calculations. The large differences of the results and the convergence behavior compared to Ref. 60 are most likely due to the use of different models for the plasmon-pole approximation. As discussed in Ref. 62, the use of a model dielectric function, which fulfills Johnson's $f$-sum rule (as the PPA of Hybertsen and Louie) ${ }^{44}$ leads to a very slow convergence of the band gap of $\mathrm{ZnO}$ with respect to the number of plane waves and unoccupied bands and gives a result, which is $1 \mathrm{eV}$ higher than obtained with the fully frequencydependent method. With the PPA of Godby and Needs on the other hand, results converge considerably faster and agree remarkably well with the frequency-dependent method.

Our results are consistent with Ref. 29 who attributed the underestimation of the gap to the starting point (PBE in their case) and also showed that the eigenvalue-sc GW method yields a band gap of $3.20 \mathrm{eV}$ in very good agreement with experiment.

The band gaps denoted GLLBSC in Table II have been obtained as the self-consistently determined Kohn-Sham band gap of a GLLBSC calculation with the estimated derivative discontinuity $\Delta_{x c}$ added. Compared to $\mathrm{G}_{0} \mathrm{~W}_{0}$, this approach yields a slightly lower accuracy compared to experiment. On the other hand, the much lower computational cost of the GLLBSC (which is comparable to LDA) makes this method very attractive for band structure calculations of large systems.

We conclude that even single-shot GW calculations with the plasmon pole approximation reproduce the experimental results to $0.2 \mathrm{eV}$ for most of the semiconductors. The largest deviations are observed for $\mathrm{ZnO}$ and $\mathrm{LiF}$ where the computed band gaps are around 1 and $0.5 \mathrm{eV}$ too small, respectively. Both of these systems have strong ionic character and LDA is presumably not a good starting point-in particular the LDA wave functions might be too delocalized. In such cases, a different starting point based on, e.g., a hybrid or LDA $+\mathrm{U}$ might yield better results although a systematic improvement seems difficult to achieve in this way. ${ }^{29}$

In Fig. 3, we compare the band structure of diamond obtained with the LDA and $\mathrm{G}_{0} \mathrm{~W}_{0} @ \mathrm{LDA}$ approximation. The valence band maximum occurs at the $\Gamma$ point and the conduction band minimum is situated along the $\Gamma-\mathrm{X}$ direction, resulting in an indirect band gap of 4.1 and $5.7 \mathrm{eV}$, respectively. We can see that the main effect of the $G_{0} W_{0}$ approximation lies in an almost constant shift of the LDA bands: Occupied bands are moved to lower energies, whereas the unoccupied

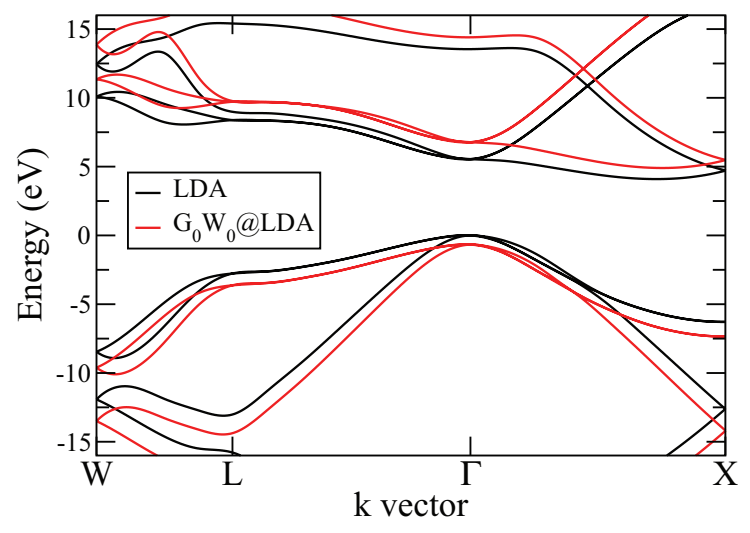

FIG. 3. (Color online) Band structure of diamond calculated with LDA (black) and $\mathrm{G}_{0} \mathrm{~W}_{0}$ (red). The bands have been interpolated by splines from a $(15 \times 15 \times 15) k$-point sampling. The band gap is indirect between the $\Gamma$ point and close to the $\mathrm{X}$ point with a value of $4.12 \mathrm{eV}$ and $5.66 \mathrm{eV}$ for LDA and $\mathrm{G}_{0} \mathrm{~W}_{0}$, respectively.

bands are shifted up. This is thus an example where the effect of $\mathrm{G}_{0} \mathrm{~W}_{0}$ is well described by a simple scissors operator.

Finally, we present the calculated band structure of gold in Fig. 4 as one example for a metallic system. The lattice parameter used for the fcc structure is $4.079 \AA$. The effect of $\mathrm{GW}$ is a small broadening of the occupied $d$ bands, with the top being shifted slightly up and the bottom down in energy. The change in the low-lying $s$ band and the unoccupied $s-p$ band are significantly larger and inhomogeneous. Our band structure agrees well with the calculations of Ref. 64 with use of the plasmon-pole approximation and exclusion of $5 \mathrm{~s}$ and $5 p$ semicore states. In Ref. 64 it was also shown that QP self-consistent GW approximation shifts the $d$ band down by $0.4 \mathrm{eV}$ relative to $\mathrm{PBE}$ in good agreement with experiments.

\section{2D STRUCTURES}

In this section we investigate the quasiparticle band structure of a two-dimensional structure composed of a single layer of hexagonal-boron nitride $(h-\mathrm{BN})$ adsorbed on $N$ layers

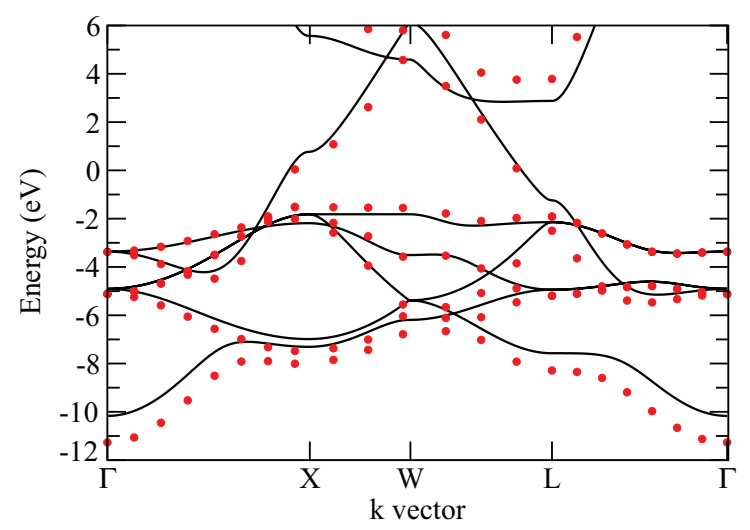

FIG. 4. (Color online) Band structure of fcc gold calculated with LDA (black lines) and $\mathrm{G}_{0} \mathrm{~W}_{0} @ \mathrm{LDA}$ with PPA (red dots). $(45 \times 45 \times 45)$ and $(15 \times 15 \times 15) k$ points have been used for LDA and GW, respectively. The bands are aligned to the respective Fermi level. 


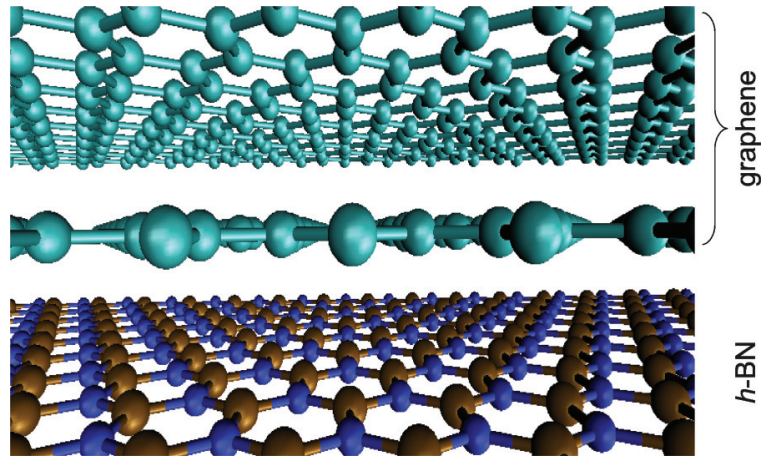

FIG. 5. (Color online) Schematic picture of the N-graphene/h-BN interface.

of graphene (as sketched in Fig. 5 for $N=2$ ). Such 2D heterostructures have recently attracted much attention due to their unique physical properties and potential application in the next-generation electronic and photonic devices. ${ }^{65-68}$

Since graphene and $h$-BN are hexagonal structures with almost the same lattice constant, $h$-BN serves as a perfect substrate for graphene. ${ }^{69}$ Based on LDA total energy calculations we find the most stable structure to be the configuration with one carbon over the $\mathrm{B}$ atom and the other carbon centered above a $h$-BN hexagon [equivalent to configuration (c) of Ref. 70] with a layer separation of $3.18 \AA$. The lattice constant is set to $2.5 \AA$ for both lattices. The calculations are performed in the same way as described in the previous section with a $k$-point sampling of $(45 \times 45)$ in the in-plane direction. Also for this system we have found that the PPA yields almost identical results to the full frequency $G_{0} W_{0}$ and therefore all calculations presented in this section have been performed with the PPA.

The importance of truncating the Coulomb potential in order to avoid spurious interaction between neighboring supercells is shown in Fig. 6 for the direct gap at the $\mathrm{K}$ point for a freestanding boron nitride monolayer. Without truncation, the gap converges very slowly with the cell size and is still $0.3 \mathrm{eV}$ below the converged value for $30 \AA$ of vacuum. Applying the truncated Coulomb potential, the band

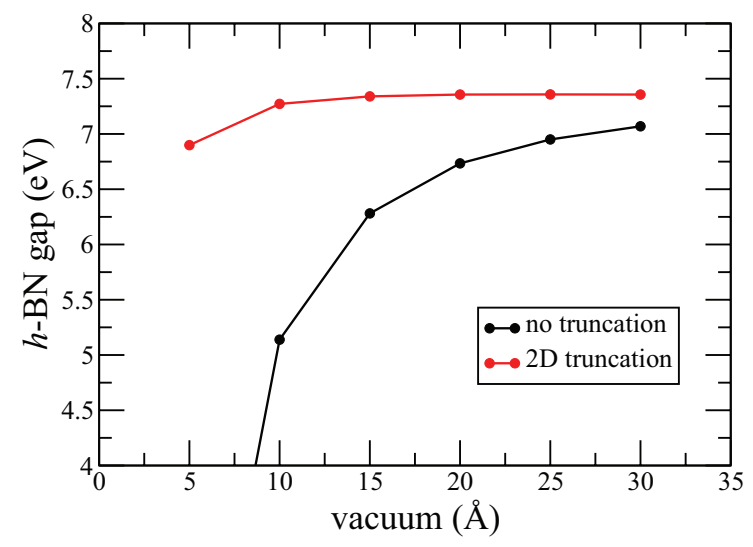

FIG. 6. (Color online) Direct $\mathrm{G}_{0} \mathrm{~W}_{0}$ band gap at the $\mathrm{K}$ point for $\mathrm{a}$ freestanding $h$-BN sheet as function of the vacuum used to separate layers in neighboring supercell with and without use of the Coulomb truncation method as described in Sec. III E.

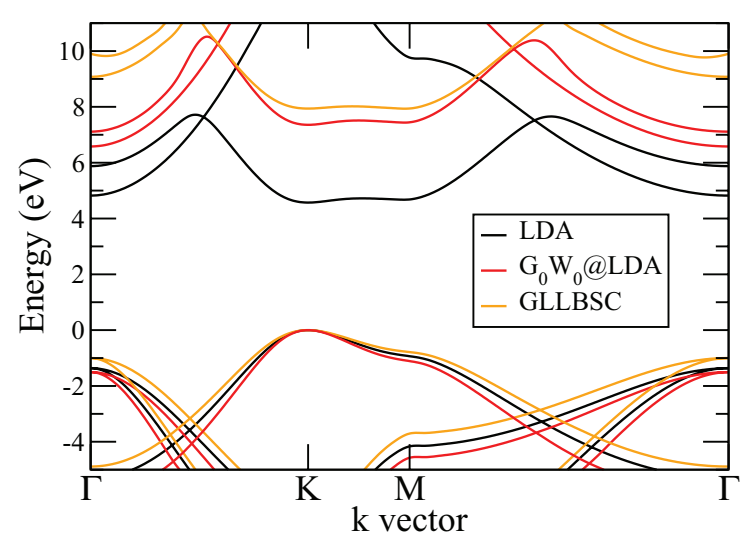

FIG. 7. (Color online) Band structure for a freestanding $h$-BN sheet. The band gap is direct at the $\mathrm{K}$ point with $\mathrm{LDA}(4.57 \mathrm{eV})$ and GLLBSC (7.94 eV) and changes to indirect between the $\mathrm{K}$ and the $\Gamma$ point for $\mathrm{G}_{0} \mathrm{~W}_{0}(7.37 \mathrm{eV})$.

gap is clearly converged already for $10 \AA$ vacuum. These observations are consistent with recent $\mathrm{G}_{0} \mathrm{~W}_{0}$ calculations for a $\mathrm{SiC}$ sheet, where the same trends were found. ${ }^{71}$

First, we summarize the most important features of the band structure calculations for the freestanding $h$-BN as shown in Fig. 7. LDA predicts a direct band gap at the K point of $4.57 \mathrm{eV}$ and an indirect $\mathrm{K}-\Gamma$ transition of $4.82 \mathrm{eV}$. With GLLBSC, the bands are shifted significantly in energy. However, the shift is not constant for the different bands, resulting in a larger increase of the gap at the $\Gamma$ point than at the $\mathrm{K}$ point. This yields $7.94 \mathrm{eV}$ and $9.08 \mathrm{eV}$ for the direct and indirect transition, respectively. The opposite is the case for $\mathrm{G}_{0} \mathrm{~W}_{0} @ \mathrm{LDA}$ calculations, which predict an indirect band gap of $6.58 \mathrm{eV}$ and a direct transition at the $\mathrm{K}$ point of $7.37 \mathrm{eV}$. These values are 0.6 and $1.0 \mathrm{eV}$ larger than the ones reported in Ref. 72 which were obtained from pseudopotential-based $\mathrm{G}_{0} \mathrm{~W}_{0} @ \mathrm{LDA}$ calculations. We note, however, that the amount of vacuum used in Ref. 72 was only $13.5 \AA$, which is not sufficient according to our results.

For the freestanding graphene (not shown), we find from the slope of the Dirac cone at the $\mathrm{K}$ point the Fermi velocity to be $0.87 \times 10^{6} \mathrm{~m} / \mathrm{s}, 0.87 \times 10^{6} \mathrm{~m} / \mathrm{s}$, and $1.17 \times 10^{6} \mathrm{~m} / \mathrm{s}$ with LDA, GLLBSC, and $\mathrm{G}_{0} \mathrm{~W}_{0}$, respectively. This is in good agreement with previous $\mathrm{G}_{0} \mathrm{~W}_{0}$ calculations, which obtained $1.15 \times 10^{6} \mathrm{~m} / \mathrm{s}$ (Ref. 73) and $1.12 \times 10^{6} \mathrm{~m} / \mathrm{s}$ (Ref. 74), respectively, and accurate magnetotransport measurements, which yielded $1.1 \times 10^{6} \mathrm{~m} / \mathrm{s}$ (Ref. 75).

The band structure of graphene on a single $h$-BN sheet is shown in Fig. 8. At a qualitative level the band structure is similar to a superposition of the band structures of the isolated systems. In particular, due to the limited coupling between the layers, the bands closest to the Fermi energy can clearly be attributed to the different layers: At the K point, the linear dispersion of the graphene bands is maintained and the second highest valence and second lowest conduction band belong to the $h$-BN. However, there are important quantitative changes. First, the slope of the Dirac cone is reduced, giving a Fermi velocity of $1.01 \times 10^{6} \mathrm{~m} / \mathrm{s}\left(0.78 \times 10^{6} \mathrm{~m} / \mathrm{s}\right)$ with $\mathrm{G}_{0} \mathrm{~W}_{0}$ (LDA). Exactly at the $\mathrm{K}$ point both LDA and $\mathrm{G}_{0} \mathrm{~W}_{0}$ predict a small gap of $50 \mathrm{meV}$. Moreover, at the $\mathrm{K}$ point, the $h$-BN gap obtained with $\mathrm{G}_{0} \mathrm{~W}_{0}$ is reduced from $7.37 \mathrm{eV}$ for the 


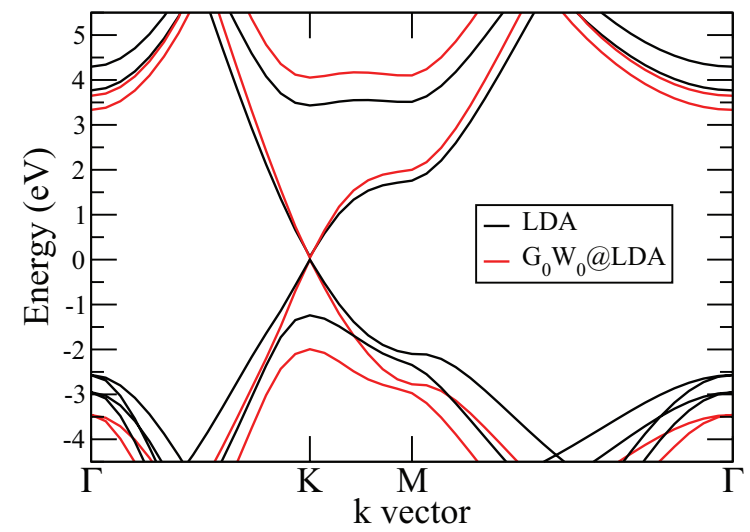

FIG. 8. (Color online) LDA and $\mathrm{G}_{0} \mathrm{~W}_{0} @ \mathrm{LDA}$ band structure for a graphene/boron nitride double layer structure. Only the two highest valence bands and the two lowest conduction bands are shown.

isolated sheet to $6.35 \mathrm{eV}$. In contrast the LDA gap is almost the same $(4.67 \mathrm{eV})$ as for the isolated $h$-BN.

To further illustrate the importance of screening effects, we calculate the dependence of the $h$-BN gap with respect to the distance between the two layers. From Fig. 9, we can see that for LDA the gap is almost constant at the value of the freestanding boron nitride. For GLLBSC, the gap is around $1.2 \mathrm{eV}$ larger but it does not change with the interlayer distance either. In contrast, GW predicts an increase of the gap with increasing distance and slowly approaches the value of the isolated system. The distance dependence of the gap is well fitted by $1 / d$ as expected from a simple image charge model. Only for small distances, the results deviate from the $1 / d$ dependence, most likely due to the formation of a chemical bond between the layers. We mention that the band gap closing due to substrate screening has been observed in previous $\mathrm{GW}$ studies of metal/semiconductor interfaces ${ }^{6,7}$ as well as for molecules on metal surfaces. ${ }^{8-11}$

In Fig. 10, the size of the $h$-BN gap is shown for a varying number of graphene layers in a $h$-BN/ $N$-graphene heterostructure. While LDA predicts a constant band gap of $h-\mathrm{BN}, \mathrm{G}_{0} \mathrm{~W}_{0}$ predicts a slight decrease of the gap with increasing number

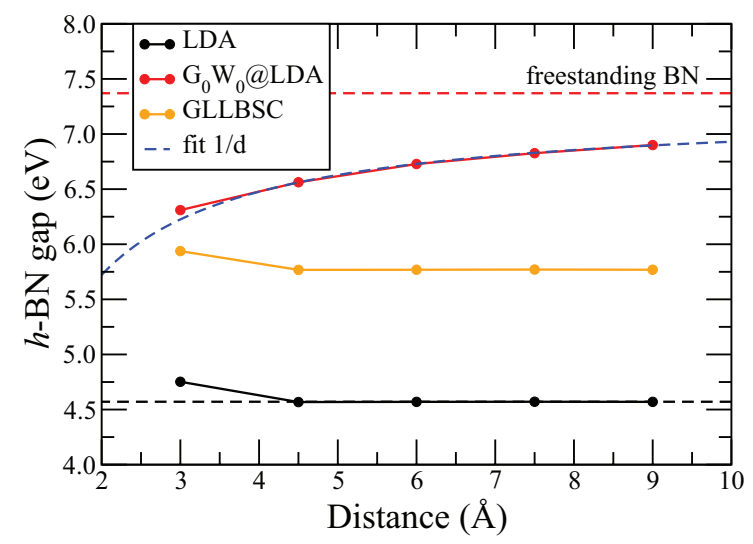

FIG. 9. (Color online) The band gap of $h$-BN at the $\mathrm{K}$ point as function of the distance to the graphene sheet (see inset). Dashed horizontal lines indicate the values for the freestanding $h$-BN, corresponding to $d \rightarrow \infty$.

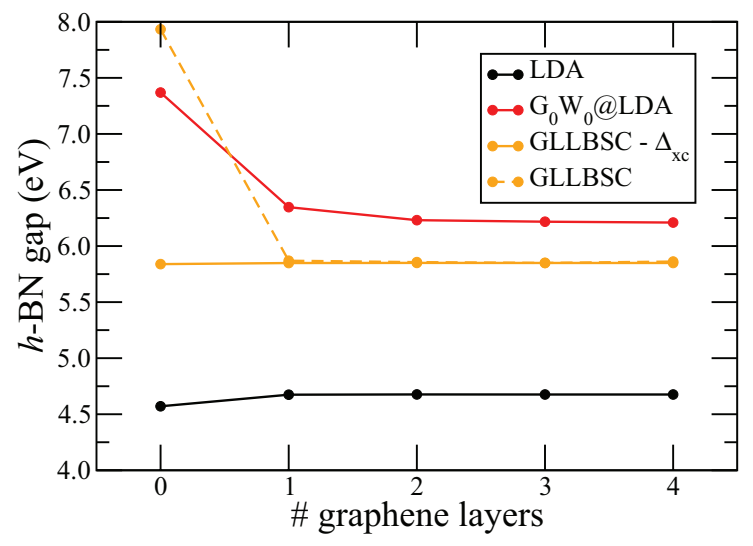

FIG. 10. (Color online) $h$-BN gap at the $\mathrm{K}$ point for different number of adsorbed graphene layers. GLLBSC results are plotted without and with the derivative discontinuity $\Delta_{x c}$.

of graphene layers due to enhanced screening. Additionally, we show the results for GLLBSC with and without the derivative discontinuity $\Delta_{x c}$ added to the Kohn-Sham gap. Due the construction of the GLLBSC, $\Delta_{x c}$ vanishes when one or more graphene layers are present because the system becomes (almost) metallic. Thus the GLLBSC gap becomes independent of the number of graphene layers, but is still close to the $\mathrm{G}_{0} \mathrm{~W}_{0}$ result.

\section{MOLECULES}

In this section, we present $\mathrm{G}_{0} \mathrm{~W}_{0}$ calculations for a set of 32 small molecules. Recently a number of high-level GW studies on molecular systems have been published..$^{25-28}$ These studies have all been performed with localized basis sets and have explored the consequences of many of the commonly made approximations related to self-consistency, starting point dependence in the $\mathrm{G}_{0} \mathrm{~W}_{0}$ approach, and treatment of core electrons. Here we use the more standard $\mathrm{G}_{0} \mathrm{~W}_{0} @ \mathrm{LDA}$ method and apply a plane-wave basis set. This is done in order to benchmark the accuracy of this scheme but also to show the universality of the present implementation in terms of the types of systems that can be treated.

Our calculations are performed in a supercell with $7 \AA$ distance between neighboring molecules in all directions. As pointed out in the previous sections, careful convergence tests are crucial in order to obtain accurate results with GW. For a plane-wave basis we have found that this is particularly important for molecules, as demonstrated in Fig. 11 for water. Here, we plot the calculated ionization potential as a function of the inverse plane-wave cutoff. Again, for each data point, the number of bands is set equal to the number of plane waves corresponding to the cutoff. Even for $E_{\text {cut }}=400 \mathrm{eV}\left(1 / E_{\text {cut }}=\right.$ $0.0025 \mathrm{eV}^{-1}$ and corresponding to more than 8000 bands), the IP is not fully converged. However, for a cutoff larger than $100 \mathrm{eV}$, the IP grows linearly with $1 / E_{\text {cut }}$ and this allows us extrapolate to the infinite cutoff (and number of empty bands) limit. ${ }^{76,77}$ In this case the converged ionization potential is $12.1 \mathrm{eV}$, which is about $0.5 \mathrm{eV}$ smaller than the experimental value. For all the molecules we have extrapolated the IP to infinite plane wave cutoff based on $\mathrm{G}_{0} \mathrm{~W}_{0}$ calculations at cutoff energies $200-400 \mathrm{eV}$. Furthermore, as found for the solids 


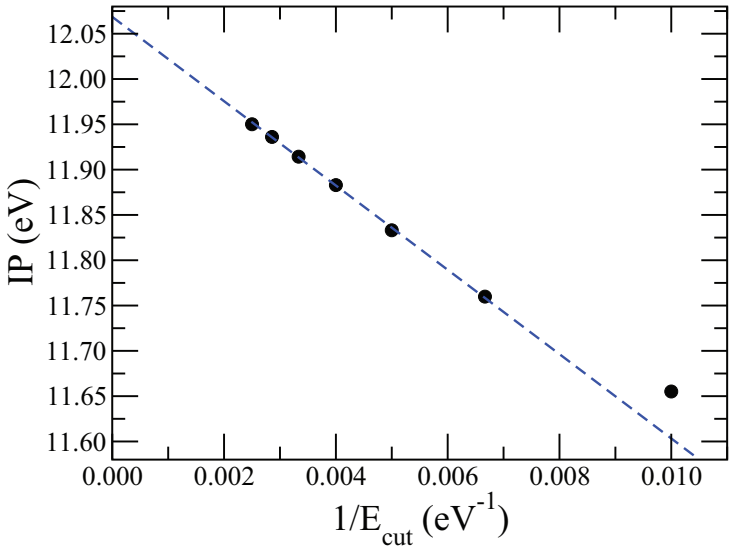

FIG. 11. (Color online) Convergence of the Ionization Potential for $\mathrm{H}_{2} \mathrm{O}$ with respect to the plane wave cutoff for $\mathrm{G}_{0} \mathrm{~W}_{0} @ \mathrm{LDA}$. The dashed line shows a linear fit of the points with $E_{\text {cut }}>100 \mathrm{eV}$ $\left(1 / E_{\text {cut }}<0.01 \mathrm{eV}^{-1}\right)$. The IP is given as the negative HOMO energy.

and the 2D systems, the plasmon-pole approximation and the fully frequency-dependent GW calculations yield very similar results with typically 0.05 to $0.1 \mathrm{eV}$ smaller IPs for the latter.

The results for all molecules are summarized and compared in Fig. 12. The LDA, PBE0 and GLLBSC calculations underestimate the IP with mean absolute errors (MAE) of $4.8,3.5$, and $2.0 \mathrm{eV}$, respectively. The opposite trend is observed for (nonselfconsistent) Hartree-Fock, which systematically overestimates the IP due to complete lack of screening. The MAE found for HF is $1.1 \mathrm{eV}$. We note that for an exact functional, according to the ionization-potential theorem, the Kohn-Sham energy of the highest occupied molecular orbital (HOMO) from DFT should be equal to the negative ionization potential. ${ }^{39}$

The $\mathrm{G}_{0} \mathrm{~W}_{0}$ results are typically around $0.5 \mathrm{eV}$ smaller than the experimental IPs, although there are a few exceptions where the calculated ionization potential is too large, and with a MAE of $0.56 \mathrm{eV}$. Recently, very similar studies have been reported for $\mathrm{G}_{0} \mathrm{~W}_{0} @ \mathrm{LDA}^{27}$ with Gaussian basis sets and $\mathrm{G}_{0} \mathrm{~W}_{0} @ \mathrm{PBE}^{28}$ in an all-electron framework using numerical atomic orbitals. Although there are differences of up to

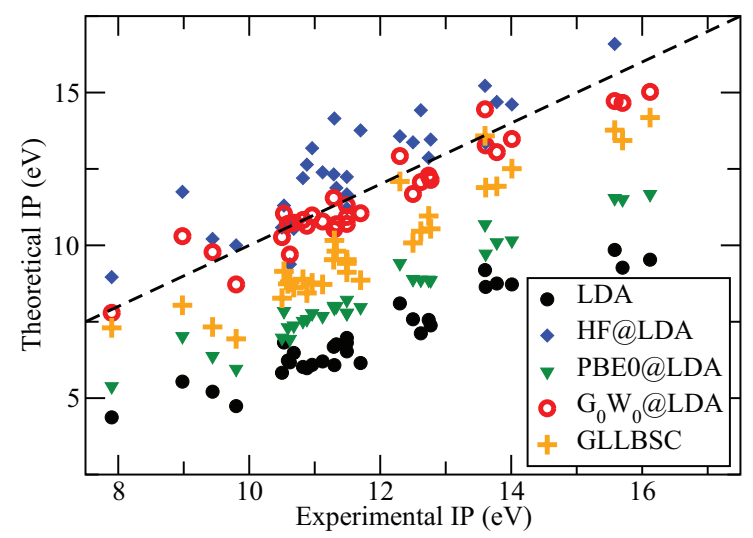

FIG. 12. (Color online) Comparison of theoretical and experimental ionization potentials. The $\mathrm{G}_{0} \mathrm{~W}_{0}$ results are obtained by applying the extrapolation scheme as explained in the text. Corresponding values are listed in Table III.

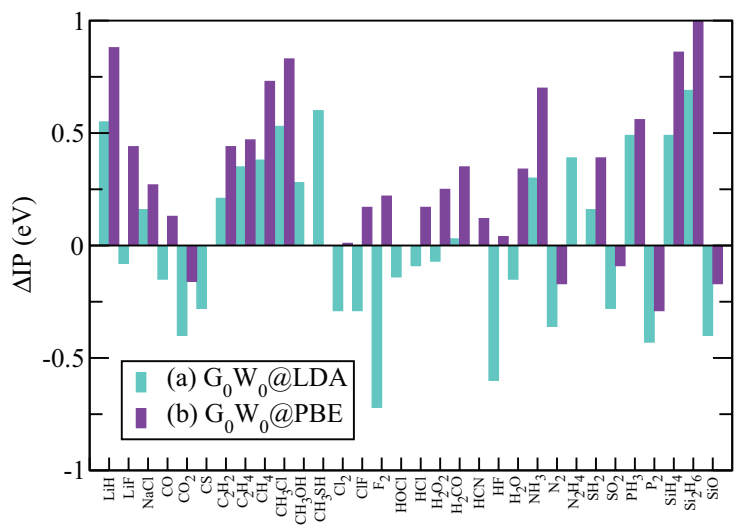

FIG. 13. (Color online) Deviations for the ionization potentials obtained with $\mathrm{G}_{0} \mathrm{~W}_{0} @ \mathrm{LDA}$ compared to (a) Ref. 27 and (b) Ref. 28. The mean deviations are 0.02 and $0.30 \mathrm{eV}$, respectively.

$0.5 \mathrm{eV}$ (both positive and negative), we find reasonable overall agreement with $0.32 \mathrm{eV}$ MAE relative to Ref. 27. The mean signed error (MSE) is only $0.02 \mathrm{eV}$. Compared to Ref. 28, our results are systematically smaller with a MAE of $0.36 \mathrm{eV}$ and a MSE of $0.30 \mathrm{eV}$. This is within the range of the accuracy of the different implementations, e.g., basis set, the PPA and the frozen core approximation applied in our calculations and the differences between LDA and PBE as starting points. A graphical comparison with these studies is shown in Fig. 13.

For detailed discussions of the role of self-consistency and other approximations we refer to Refs. 25,27,28, and 78 .

\section{CONCLUSION}

We have presented a plane-wave implementation of the single-shot $\mathrm{G}_{0} \mathrm{~W}_{0}$ approximation within the GPAW projector augmented wave method code. The method has been applied to the calculation of quasiparticle band structures and energy levels in bulk crystals, 2D materials, and molecules, respectively. Particular attention has been paid to the convergence of the calculations with respect to the plane-wave cutoff and the number of unoccupied bands. While for all extended systems the value of the band gap was found to be converged at around $200 \mathrm{eV}$, the ionization potentials of the molecules required significantly higher cutoffs. In these cases, the data points were fit linearly to $1 / E_{\text {cut }}$, allowing to extrapolate to infinite number of bands. For all calculations, the plasmon-pole approximation and the use of full frequency dependence of the dielectric function and the screened potential give very similar results. With these two observations, the computational demands can be drastically reduced without losing accuracy.

For the bulk semiconductors, we found good agreement with experimental results with a mean absolute error (MAE) of $0.2 \mathrm{eV}$. However, in the special case of zinc oxide and for the large gap insulators, the calculated band gaps were underestimated by $0.5-1 \mathrm{eV}$. These errors are most likely due to the lack of self-consistency and/or the quality of the LDA starting point used in our calculations. Similar conclusions apply to the 32 small molecules where the ionization potentials obtained from $\mathrm{G}_{0} \mathrm{~W}_{0} @ \mathrm{LDA}$ were found to underestimate the experimental values by around $0.5 \mathrm{eV}$ on average. The important role of screening for the quasiparticle band structure 
TABLE III. Calculated and experimental ionization potentials. All energies are in eV. Last row shows the mean absolute error (MAE) with respect to experiments. Experimental data taken from Ref. 79.

\begin{tabular}{|c|c|c|c|c|c|c|}
\hline Molecule & LDA & HF@LDA & PBE0@LDA & GLLBSC & $\mathrm{G}_{0} \mathrm{~W}_{0} @ \mathrm{LDA}$ & Experiment \\
\hline $\mathrm{LiH}$ & 4.37 & 8.96 & 5.38 & 7.30 & 7.79 & 7.90 \\
\hline $\mathrm{LiF}$ & 6.08 & 14.15 & 7.95 & 10.16 & 10.53 & 11.30 \\
\hline $\mathrm{NaCl}$ & 4.74 & 10.00 & 5.95 & 6.94 & 8.72 & 9.80 \\
\hline $\mathrm{CO}$ & 8.72 & 14.61 & 10.15 & 12.51 & 13.48 & 14.01 \\
\hline $\mathrm{CO}_{2}$ & 8.75 & 14.69 & 10.09 & 11.93 & 13.05 & 13.78 \\
\hline $\mathrm{CS}$ & 6.76 & 11.88 & 8.00 & 9.81 & 10.69 & 11.33 \\
\hline $\mathrm{C}_{2} \mathrm{H}_{2}$ & 6.81 & 11.21 & 7.79 & 9.41 & 11.22 & 11.49 \\
\hline $\mathrm{C}_{2} \mathrm{H}_{4}$ & 6.48 & 10.54 & 7.37 & 8.62 & 10.74 & 10.68 \\
\hline $\mathrm{CH}_{4}$ & 9.19 & 15.22 & 10.68 & 13.58 & 14.45 & 13.60 \\
\hline $\mathrm{CH}_{3} \mathrm{Cl}$ & 6.68 & 12.32 & 8.01 & 9.53 & 11.55 & 11.29 \\
\hline $\mathrm{CH}_{3} \mathrm{OH}$ & 6.09 & 13.18 & 7.77 & 8.77 & 10.98 & 10.96 \\
\hline $\mathrm{CH}_{3} \mathrm{SH}$ & 5.21 & 10.21 & 6.37 & 7.33 & 9.78 & 9.44 \\
\hline $\mathrm{Cl}_{2}$ & 6.53 & 11.67 & 7.77 & 9.12 & 10.93 & 11.49 \\
\hline $\mathrm{ClF}$ & 7.38 & 13.46 & 8.85 & 10.54 & 12.14 & 12.77 \\
\hline $\mathrm{F}_{2}$ & 9.27 & 18.44 & 11.50 & 13.43 & 14.66 & 15.70 \\
\hline $\mathrm{HOCl}$ & 6.20 & 12.39 & 7.68 & 8.72 & 10.78 & 11.12 \\
\hline $\mathrm{HCl}$ & 7.56 & 12.86 & 8.87 & 10.96 & 12.28 & 12.74 \\
\hline $\mathrm{H}_{2} \mathrm{O}_{2}$ & 6.15 & 13.76 & 7.97 & 8.86 & 11.05 & 11.70 \\
\hline $\mathrm{H}_{2} \mathrm{CO}$ & 5.98 & 12.64 & 7.58 & 8.44 & 10.64 & 10.88 \\
\hline $\mathrm{HCN}$ & 8.64 & 13.35 & 9.72 & 11.89 & 13.27 & 13.61 \\
\hline $\mathrm{HF}$ & 9.53 & 18.29 & 11.67 & 14.18 & 15.02 & 16.12 \\
\hline $\mathrm{H}_{2} \mathrm{O}$ & 7.12 & 14.42 & 8.87 & 10.46 & 12.07 & 12.62 \\
\hline $\mathrm{NH}_{3}$ & 6.02 & 12.20 & 7.52 & 8.89 & 10.83 & 10.82 \\
\hline $\mathrm{N}_{2}$ & 9.85 & 16.59 & 11.54 & 13.77 & 14.72 & 15.58 \\
\hline $\mathrm{N}_{2} \mathrm{H}_{4}$ & 5.54 & 11.75 & 7.02 & 8.04 & 10.30 & 8.98 \\
\hline $\mathrm{SH}_{2}$ & 5.83 & 10.58 & 6.97 & 8.27 & 10.27 & 10.50 \\
\hline $\mathrm{SO}_{2}$ & 7.58 & 13.37 & 8.89 & 10.08 & 11.68 & 12.50 \\
\hline $\mathrm{PH}_{3}$ & 6.23 & 10.77 & 7.31 & 8.74 & 10.70 & 10.59 \\
\hline $\mathrm{P}_{2}$ & 6.17 & 9.38 & 6.93 & 8.80 & 9.70 & 10.62 \\
\hline $\mathrm{SiH}_{4}$ & 8.10 & 13.57 & 9.41 & 12.09 & 12.92 & 12.30 \\
\hline $\mathrm{Si}_{2} \mathrm{H}_{6}$ & 6.82 & 11.30 & 7.84 & 9.15 & 11.04 & 10.53 \\
\hline $\mathrm{SiO}$ & 6.97 & 12.24 & 8.21 & 9.53 & 10.70 & 11.49 \\
\hline$\overline{\mathrm{MAE}}$ & 4.84 & 1.11 & 3.46 & 1.83 & 0.56 & \\
\hline
\end{tabular}

was illustrated by the case of a $2 \mathrm{D}$ graphene/boron-nitride heterojunction. For this system, we found a truncation of the Coulomb potential to be crucial in periodic supercell calculations.

The $\mathrm{G}_{0} \mathrm{~W}_{0}$ results were compared to band structures obtained with Hartree-Fock, the PBE0 hybrid, and the GLLBSC potential. While Hartree-Fock and PBE0 yield overall poor results, the computationally efficient GLLBSC results were found to be in surprisingly good agreement with $\mathrm{G}_{0} \mathrm{~W}_{0}$ for the band gaps of semiconductors, while the ionization potentials of molecules were found to be $1.5 \mathrm{eV}$ lower on average.

\section{ACKNOWLEDGMENTS}

We would like to thank Jun Yan and Jens Jørgen Mortensen for useful discussions and assistance with the coding. The authors acknowledge support from the Danish Council for Independent Research's Sapere Aude Program through Grant No. 11-1051390. The Center for Nanostructured Graphene is sponsored by the Danish National Research Foundation. The Catalysis for Sustainable Energy (CASE) initiative is funded by the Danish Ministry of Science, Technology and Innovation.

\footnotetext{
*falco.hueser@fysik.dtu.dk

†thygesen@fysik.dtu.dk

${ }^{1}$ P. Hohenberg and W. Kohn, Phys. Rev. 136, B864 (1964).

${ }^{2}$ W. Kohn and L. J. Sham, Phys. Rev. 140, A1133 (1965).

${ }^{3}$ R. W. Godby, M. Schlüter, and L. J. Sham, Phys. Rev. B 37, 10159 (1988).

${ }^{4}$ F. Bechstedt, F. Fuchs, and G. Kresse, Phys. Status Solidi B 246, 1877 (2009).
}

${ }^{5}$ L. Hedin, Phys. Rev. 139, A796 (1965).

${ }^{6}$ J. P. A. Charlesworth, R. W. Godby, and R. J. Needs, Phys. Rev. Lett. 70, 1685 (1993).

${ }^{7}$ J. C. Inkson, J. Phys. C 6, 1350 (1973).

${ }^{8}$ J. B. Neaton, M. S. Hybertsen, and S. G. Louie, Phys. Rev. Lett. 97, 216405 (2006).

${ }^{9}$ J. M. Garcia-Lastra, C. Rostgaard, A. Rubio, and K. S. Thygesen, Phys. Rev. B 80, 245427 (2009). 
${ }^{10}$ K. S. Thygesen and A. Rubio, Phys. Rev. Lett. 102, 046802 (2009).

${ }^{11}$ C. Freysoldt, P. Rinke, and M. Scheffler, Phys. Rev. Lett. 103, 056803 (2009).

${ }^{12}$ W. G. Aulbur, L. Jönsson, and J. W. Wilkins, Solid State Phys. 54, 1 (2000).

${ }^{13}$ F. Aryasetiawan and O. Gunnarsson, Rep. Prog. Phys. 61, 237 (1998).

${ }^{14}$ G. Onida, L. Reining, and A. Rubio, Rev. Mod. Phys. 74, 601 (2002).

${ }^{15}$ S. Albrecht, L. Reining, R. Del Sole, and G. Onida, Phys. Rev. Lett. 80, 4510 (1998).

${ }^{16}$ E. E. Salpeter and H. A. Bethe, Phys. Rev. 84, 1232 (1951).

${ }^{17}$ M. Rohlfing and S. G. Louie, Phys. Rev. B 62, 4927 (2000).

${ }^{18}$ J. Yan, K. W. Jacobsen, and K. S. Thygesen, Phys. Rev. B 86, 045208 (2012).

${ }^{19}$ K. S. Thygesen and A. Rubio, J. Chem. Phys. 126, 091101 (2007).

${ }^{20}$ K. S. Thygesen and A. Rubio, Phys. Rev. B 77, 115333 (2008).

${ }^{21}$ P. Darancet, A. Ferretti, D. Mayou, and V. Olevano, Phys. Rev. B 75, 075102 (2007).

${ }^{22}$ M. Strange, C. Rostgaard, H. Häkkinen, and K. S. Thygesen, Phys. Rev. B 83, 115108 (2011).

${ }^{23}$ M. Strange and K. S. Thygesen, Beilstein J. Nanotechnol. 2, 746 (2011).

${ }^{24}$ G. Strinati, H. J. Mattausch, and W. Hanke, Phys. Rev. B 25, 2867 (1982).

${ }^{25}$ C. Rostgaard, K. W. Jacobsen, and K. S. Thygesen, Phys. Rev. B 81, 085103 (2010).

${ }^{26}$ X. Blase, C. Attaccalite, and V. Olevano, Phys. Rev. B 83, 115103 (2011).

${ }^{27}$ F. Bruneval and M. A. L. Marques, J. Chem. Theory Comput. 9, 324 (2013).

${ }^{28}$ F. Caruso, P. Rinke, X. Ren, M. Scheffler, and A. Rubio, Phys. Rev. B 86, 081102(R) (2012).

${ }^{29}$ M. Shishkin and G. Kresse, Phys. Rev. B 75, 235102 (2007).

${ }^{30}$ S. V. Faleev, M. van Schilfgaarde, and T. Kotani, Phys. Rev. Lett. 93, 126406 (2004).

${ }^{31}$ M. P. Surh, S. G. Louie, and M. L. Cohen, Phys. Rev. B 43, 9126 (1991).

${ }^{32}$ T. Kotani, M. van Schilfgaarde, S. V. Faleev, and A. Chantis, J. Phys.: Condens. Matter 19, 365236 (2007).

${ }^{33}$ M. Strange and K. S. Thygesen, Phys. Rev. B 86, 195121 (2012).

${ }^{34}$ J. Enkovaara, C. Rostgaard, J. J. Mortensen, J. Chen, M. Dulak, L. Ferrighi, J. Gavnholt, C. Glinsvad, V. Haikola, H. A. Hansen et al., J. Phys.: Condens. Matter 22, 253202 (2010).

${ }^{35}$ P. E. Blöchl, Phys. Rev. B 50, 17953 (1994).

${ }^{36}$ P. E. Blöchl, C. J. Först, and J. Schimpl, Bull. Mater. Sci. 26, 33 (2003).

${ }^{37}$ R. W. Godby and R. J. Needs, Phys. Rev. Lett. 62, 1169 (1989).

${ }^{38}$ O. Gritsenko, R. van Leeuwen, E. van Lenthe, and E. J. Baerends, Phys. Rev. A 51, 1944 (1995).

${ }^{39}$ J. P. Perdew, R. G. Parr, M. Levy, and J. L. Balduz, Jr., Phys. Rev. Lett. 49, 1691 (1982).

${ }^{40} \mathrm{H}$. Bruus and K. Flensberg, Many-Body Quantum Theory in Condensed Matter Physics - An Introduction (Oxford University Press, Oxford, 2004).

${ }^{41}$ M. Shishkin and G. Kresse, Phys. Rev. B 74, 035101 (2006).

${ }^{42}$ J. Yan, J. J. Mortensen, K. W. Jacobsen, and K. S. Thygesen, Phys. Rev. B 83, 245122 (2011).
${ }^{43}$ A. Sorouri, W. M. Foulkes, and N. D. Hine, J. Chem. Phys. 124, 064105 (2006)

${ }^{44}$ M. S. Hybertsen and S. G. Louie, Phys. Rev. B 34, 5390 (1986).

${ }^{45}$ C. A. Rozzi, D. Varsano, A. Marini, E. K. U. Gross, and A. Rubio, Phys. Rev. B 73, 205119 (2006).

${ }^{46}$ S. Ismail-Beigi, Phys. Rev. B 73, 233103 (2006).

${ }^{47}$ C. Freysoldt, P. Eggert, P. Rinke, A. Schindlmayr, R. W. Godby, and M. Scheffler, Comput. Phys. Commun. 176, 1 (2007).

${ }^{48}$ C. Freysoldt, P. Eggert, P. Rinke, A. Schindlmayr, and M. Scheffler, Phys. Rev. B 77, 235428 (2008).

${ }^{49}$ M. Kuisma, J. Ojanen, J. Enkovaara, and T. T. Rantala, Phys. Rev. B 82, 115106 (2010).

${ }^{50}$ I. E. Castelli, T. Olsen, S. Datta, D. D. Landis, S. Dahl, K. S. Thygesen, and K. W. Jacobsen, Energy Environ. Sci. 5, 5814 (2012).

${ }^{51}$ I. E. Castelli, D. D. Landis, S. Dahl, K. S. Thygesen, I. Chorkendorff, T. F. Jaramillo, and K. W. Jacobsen, Energy Environ. Sci. 5, 9034 (2012).

${ }^{52}$ W. Kang and M. S. Hybertsen, Phys. Rev. B 82, 195108 (2010).

${ }^{53}$ F. Bruneval, N. Vast, and L. Reining, Phys. Rev. B 74, 045102 (2006).

${ }^{54}$ T. Kotani and M. van Schilfgaarde, Solid State Commun. 121, 461 (2002).

${ }^{55}$ J. Harl, L. Schimka, and G. Kresse, Phys. Rev. B 81, 115126 (2010).

${ }^{56}$ M. Usuda, N. Hamada, T. Kotani, and M. van Schilfgaarde, Phys. Rev. B 66, 125101 (2002).

${ }^{57}$ H. Dixit, R. Saniz, D. Lamoen, and B. Partoens, J. Phys.: Condens. Matter 22, 125505 (2010).

${ }^{58}$ P. Rinke, A. Qteish, J. Neugebauer, C. Freysoldt, and M. Scheffler, New J. Phys. 7, 126 (2005).

${ }^{59}$ F. Fuchs, J. Furthmüller, F. Bechstedt, M. Shishkin, and G. Kresse, Phys. Rev. B 76, 115109 (2007).

${ }^{60}$ B.-C. Shih, Y. Xue, P. Zhang, M. L. Cohen, and S. G. Louie, Phys. Rev. Lett. 105, 146401 (2010).

${ }^{61}$ C. Friedrich, M. C. Müller, and S. Blügel, Phys. Rev. B 83, 081101(R) (2011).

${ }^{62}$ M. Stankovski, G. Antonius, D. Waroquiers, A. Miglio, H. Dixit, K. Sankaran, M. Giantomassi, X. Gonze, M. Côté, and G.-M. Rignanese, Phys. Rev. B 84, 241201(R) (2011).

${ }^{63}$ I. Vurgaftman, J. R. Meyer, and L. R. Ram-Mohan, J. Appl. Phys. 89, 5815 (2001).

${ }^{64}$ T. Rangel, D. Kecik, P. E. Trevisanutto, G.-M. Rignanese, H. Van Swygenhoven, and V. Olevano, Phys. Rev. B 86, 125125 (2012).

${ }^{65}$ L. A. Ponomarenko, A. K. Geim, A. A. Zhukov, R. Jalil, S. V. Morozov, K. S. Novoselov, I. V. Grigorieva, E. H. Hill, V. V. Cheianov, V. I. Fal'ko et al., Nature Phys. 7, 958 (2011).

${ }^{66} \mathrm{H}$. Wang, T. Taychatanapat, A. Hsu, K. Watanabe, T. Taniguchi, P. Jarillo-Herrero, and T. Palacios, IEEE Electron Device Lett. 32, 1209 (2011).

${ }^{67}$ S. J. Haigh, A. Gholinia, R. Jalil, S. Romani, L. Britnell, D. C. Elias, K. S. Novoselov, L. A. Ponomarenko, A. K. Geim, and R. Gorbachev, Nature Mater. 11, 764 (2012).

${ }^{68}$ L. Britnell, R. V. Gorbachev, R. Jalil, B. D. Belle, F. Schedin, A. Mishchenko, T. Georgiou, M. I. Katsnelson, L. Eaves, S. V. Morozov et al., Science 335, 947 (2012).

${ }^{69}$ R. Decker, Y. Wang, V. W. Brar, W. Regan, H.-Z. Tsai, Q. Wu, W. Gannett, A. Zettl, and M. F. Crommie, Nano Lett. 11, 2291 (2011).

${ }^{70}$ G. Giovannetti, P. A. Khomyakov, G. Brocks, P. J. Kelly, and J. van den Brink, Phys. Rev. B 76, 073103 (2007). 
${ }^{71}$ H. C. Hsueh, G. Y. Guo, and S. G. Louie, Phys. Rev. B 84, 085404 (2011).

${ }^{72}$ X. Blase, A. Rubio, S. G. Louie, and M. L. Cohen, Phys. Rev. B 51, 6868 (1995).

${ }^{73}$ L. Yang, J. Deslippe, C.-H. Park, M. L. Cohen, and S. G. Louie, Phys. Rev. Lett. 103, 186802 (2009).

${ }^{74}$ P. E. Trevisanutto, C. Giorgetti, L. Reining, M. Ladisa, and V. Olevano, Phys. Rev. Lett. 101, 226405 (2008).

${ }^{75}$ Y. Zhang, Y.-W. Tan, H. L. Stormer, and P. Kim, Nature (London) 438, 201 (2005).

${ }^{76}$ W. Kang and M. S. Hybertsen, Phys. Rev. B 82, 085203 (2010).
${ }^{77}$ P. Umari, X. Qian, N. Marzari, G. Stenuit, L. Giacomazzi, and S. Baroni, Phys. Status Solidi B 248, 527 (2011).

${ }^{78}$ A. Stan, N. E. Dahlen, and R. van Leeuwen, J. Chem. Phys. 130, 114105 (2009).

${ }^{79}$ NIST Computational Chemistry Comparison and Benchmark Database, NIST Standard Reference Database Number 101, Release 15b, Aug 2011, edited by Russell D. Johnson III; http://cccbdb.nist.gov/

${ }^{80}$ With the use of $c_{\phi}^{\dagger}=\int d \mathbf{r} \phi^{*}(\mathbf{r}) \hat{\Psi}^{\dagger}(\mathbf{r})$.

${ }^{81}$ The dual basis functions are in fact the eigenvectors of the adjoint operator $\left[H_{0}+\Sigma(z)\right]^{\dagger}$. 